\title{
Apparent Experimental Confirmation of Pseudoprotons and Their Application to New Clean Nuclear Energies
}

\author{
Ruggero Maria Santilli* \\ Thunder Energies Corporation, 1444 Rainville Rd, Tarpon Springs, Fl, 34689, U.S.A. \\ * Corresponding author. Tel.: 727-688-3992; email: research@thunder-energies.com \\ Manuscript submitted May 9, 2018; accepted December 20, 2018. \\ doi: 10.17706/ijapm.2019.9.2.72-100
}

\begin{abstract}
In preceding papers initiated in the 1980s at Harvard University under DOE support, the author presented evidence of the laboratory synthesis of the neutron from an electron and a proton, as well as the prediction of a second synthesis of an electron and, this time, a neutron, resulting in a new nucleon known as the pseudoproton, with predicted characteristics: negative charge, spin $1 / 2$, charge radius and mass essentially those of the neutron, and mean life of the order of seconds, thus suitable for applications. In this paper, we present apparently for the first time: two different reactors predicted to synthesize pseudoprotons jointly with other particles; pseudoproton irradiation of natural elements; experimental evidence from two U. S. spectroscopic laboratories supporting the existence of the pseudoproton; the pseudoproton capability of resolving the historical Coulomb repulsion between nuclei thanks to its negative charge; and the possible existence of new nuclear energies without the emission of harmful radiations and without the release of radioactive waste caused by pseudoproton irradiation of a selected number of light natural elements.
\end{abstract}

Key words: Neutrons, neutroids, pseudoprotons, protoids.

\section{The Synthesis of Neutrons and Neutroids from the Hydrogen}

\subsection{The Synthesis of the Neutron}

H. Rutherford [1] suggested in 1910 that the hydrogen atom in the core of stars is "compressed" into a new neutral particle which he called the neutron

$$
p^{+}+e^{-} \rightarrow n
$$

The existence of the neutron was experimentally confirmed by J. Chadwick [2] in 1932.

E. Fermi [3] noted that the above reaction violates the principle of conservation of the angular momentum and therefore, submitted the hypothesis of the emission of a neutral and massless particle which he called neutrino (meaning "little neutron" in Italian)

$$
p^{+}+e^{-} \rightarrow n+v \text {. }
$$

Attempts at the laboratory synthesis of the neutron from the hydrogen were initiated in the 1950's [4], although none of them achieved published results due to the impossibility of reducing the strong interactions of the neutron to pure electromagnetic contributions.

Special attempts for the laboratory synthesis of the neutron were done in the 1960's by the Italian priest-physicist Don Carlo Borghi and his colleagues [5] via a reactor containing a hydrogen gas at $15 p s i$ 
kept ionized by a $500 \mathrm{~V}$ DC arc with $10 \mathrm{~mA}$. The neutron synthesis (2) was assumed to be caused by microwaves with $10^{10} \mathrm{~s}^{-1}$.

Ref. [5] presents considerable evidence of nuclear transmutations occurred in various natural elements placed outside the reactor, which transmutations were interpreted as being due to a flux of neutrons that could only originate from the interior of the reactor, although neutrons were not directly detected in Ref. [5].

The author conducted systematic, mathematical, theoretical and experimental studies on the synthesis of the neutron from the hydrogen beginning the late 1970's while he was at Harvard University under DOE support [6]-[38] (see [39]-[61] for additional studies). The main difficulty was that the restenergy of the neutron is bigger than the sum of the rest energies of the proton and the electron,

$$
\begin{gathered}
E_{p}=938.272 \mathrm{MeV}, \quad E_{e}=0.511 \mathrm{MeV}, \quad E_{n}=939.565 \mathrm{MeV}, \\
E_{n}-\left(E_{p}+E_{e}\right)=0.782 \mathrm{MeV}>0 .
\end{gathered}
$$

Under the above conditions, the Schrodinger equation of quantum mechanics is exactly valid for an electron orbiting in vacuum around the proton in the hydrogen atom (conditions known as exterior dynamical problems), but said equation does not yield physically consistent results for the same electron when "compressed" within the hyperdense medium inside the proton (conditions known as interior dynamical problems) due to numerous reasons, including: the need for a "positive binding energy" with ensuing "mass excess" which are anathema for quantum mechanics; the emergence in interior dynamical problems of non-linear, non-local and non-Hamiltonian interactions due to total mutual penetration of particle, which interactions are outside any representational capability by quantum mechanics; and other reasons.

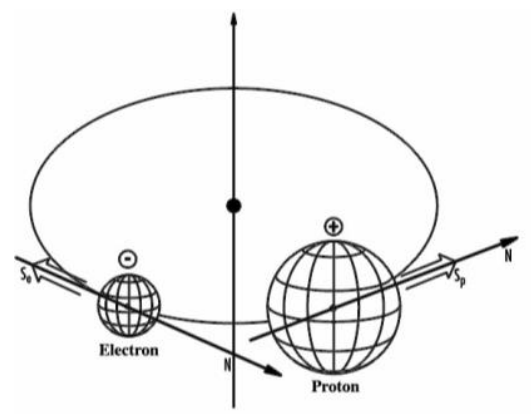

Fig. 1. A schematic view of the ionization of the hydrogen atom into protons and electrons and their orientation along a magnetic line caused by a rapid DC arc represented by the vertical line.

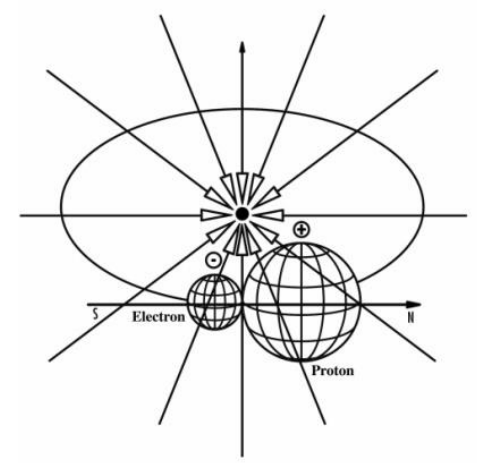

Fig. 2. A schematic view of the compression of the electron and the proton caused by the disconnection of a rapid DC arc in addition to their natural Coulomb attraction. 
Similarly, the Dirac's equation of relativistic quantum mechanics remains valid for the structure of the hydrogen atom, although said equation becomes inapplicable (rather than "violated") for the "compression" of the same atom into the neutron, not only be- cause of the need to achieve an "excess mass," but also due to the inevitable emergence of non-linear, non-local and non-Hamiltonian effects beyond any hope of consistent representation by relativistic quantum mechanics.

In particular, the inability to achieve a quantitative representation of the neutron syn- thesis resulted as beingdue to the insufficiency of the mathematics of 20th centurysciences (rather than the physical laws), due to the known sole representation of point particles under which a point-like electron cannot possibly be "compressed" inside a point-like proton.

Due to the above insufficiencies, the author had no other option than that of con- structing the foundations of new mathematics, today known as isomathematics [6]-[9] (see independent studies [10]-[13] and large references quoted therein) for the representation of extended particles in conditions of mutual penetration thus being under generally non- linear, non-local and non-Hamiltonian interactions.

Following the achievement of a consistent formulation of the novel isomathematics, the author constructed the foundations of a covering of quantum mechanics known as hadronic mechanics [6], [7] whose simplest branch characterized by isomathematics is known as isomechanics [14]-[20] (see also independent studies [21]-[25] and large literature quoted therein). The new isomathematics and isomechanics were then subjected to experimental verifications in various fields [19].

The prefix "iso" in isomathematics and isomechanics was suggested [6], [7] in its Greek sense of preserving the original axioms and merely presenting broader realizations. There- fore, the axiomatic consistency of the new sciences was assured by the fact that they coincide with 20 th century sciences at the abstract, realization-free level.

Following the achievement of consistent foundations of isomathematics and isomechanics, the author was finally able to achieve a numerically exact and time invariant representation at the non-relativistic [26]-[29] and relativistic level [29], [30] of all characteristics of the neutron in its synthesis from the hydrogen atoms, including the representation of: rest energy, mean life, charge radius, charge, spin, anomalous magnetic moment, parity and spontaneous decay (when isolated).

These studies essentially established that the strong interactions of the neutrons are primarily due to the non-linear, non-local and non-Hamiltonian interactions occurring under conditions of mutual penetration of extended particles. In fact, said interactions have the same range as that of strong interactions, are strongly attractive for particles in singlet coupling, and are charge independent because reducible to an attractive Hulten potential which is known to "absorb" Coulomb potentials at short distances irrespectively of whether attractive or repulsive (see Sect. 5 of Refs. [6] and subsequent works).

These studies clarified that the lack of representation of strong interactions in all pre- ceding works [4], [5] was due to their sole use of 20th century sciences and their inherent abstraction of particles as being point-like, resulting in purely potential interactions.

Following the achievement of a mathematical and theoretical understanding of the neutron synthesis, the author conducted systematic experimental tests for the laboratory synthesis of the neutron from a hydrogen gas [31]-[35]. These tests resulted in the industrial production and sale by the U.S. publicly traded company Thunder Energies Corporation (www.thunder-energies.com) of Directional Neutron Sources (DNS) used in the experimental verifications presented in this paper (see Section 4.2 for a description).

These experimental tests essentially established that the neutron can be synthesized via a rapid DC discharged between a pair of carbon electrodes submerged within a hydrogen gas provided that said discharge has characteristics over the threshold value of $3 \mathrm{kV}$ and $300 \mathrm{~J}$. Below these values it appears that the DC arc does not possess sufficient energy to supply the missing $0.782 \mathrm{MeV}$ needed for the neutron 
synthesis.

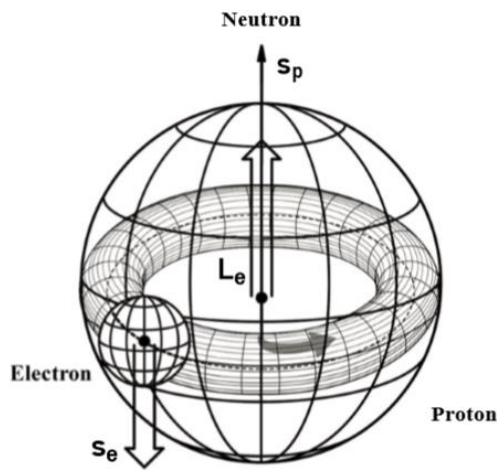

Fig. 3. A conceptual rendering of Rutherford's [1] synthesis of the neutron in the core of stars via the "compression" of the electron within the hyperdense proton in singlet coupling.

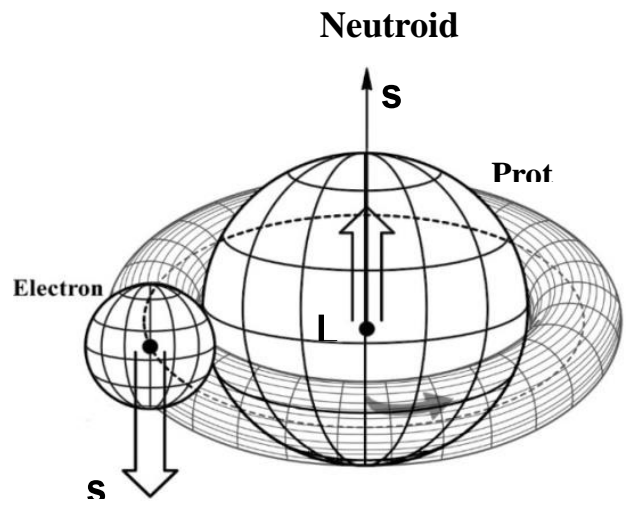

Fig. 4. A conceptual rendering of the intermediate state prior to the full synthesis of the neutron known as "neutroid" ]26 in singlet coupling at $1 \mathrm{fm}$ mutual distance which is solely possible under the strongly-attractive contact interactions of the isomechanical branch of hadronic mechanics.

A recent presentation of the synthesis of the neutron from a hydrogen gas via a submerged rapid DC discharge is available in Ref. [35]. Refs. [36], [37] provide introductory general reviews. Monograph [38] provides a comprehensive review of the mathematical, theoretical and experimental aspects of the synthesis of the neutrons from the hydrogen. None of the particles synthesized in this paper can be consistently treated with quantum mechanics and its inherent point-like abstraction of particles. A knowledge of isomathematics and isomechanics (hereon collectively referred to as New Sciences) as presented in [20], [38] is essential for the understanding of this paper since said sciences are used for the quantitative representation of all composite particles studied in this paper.

\subsection{The Synthesis of Neutroids}

The main steps of the synthesis of particles from the hydrogen studied in this paper can be summarized as follows: the DC discharge between submerged carbon electrodes ionizes the hydrogen resulting in a plasma composed by protons and electrons which are aligned along a local magnetic line of the arc with opposing spins, charges and magnetic moments (see Fig. 1 for a conceptual rendering).

For reasons not entirely understood, the disconnection of the DC discharge "compresses" the proton and the electron toward the symmetry axis (Fig. 2). In the event of values of the DC arc below the indicated threshold values, the proton and the electron are bounded into a state at $1 \mathrm{fm}$ mutual distance called neutroid [31], [32] (Fig. 3) 


$$
p_{\uparrow}^{+}+e_{\downarrow}^{-} \rightarrow \tilde{n},
$$

With the following characteristics: charge $q=0$; spin $s=0$ (under the assumption that the orbital motion of the electron is in its hadronic ground state); radius essentially the same as that of the neutron; mean-life estimated to be of $9 s$; rest energy (for $c=1$ )

$$
\begin{gathered}
E_{\tilde{n}=m_{p}+m_{e}-} B E=938.2721+0.511-0.009 \mathrm{MeV}= \\
=938.7741 \mathrm{MeV}=1.008091 \mathrm{u},
\end{gathered}
$$

where the binding energy is assumed to be of sole Coulomb character since contact non- Hamiltonian interactions have no potential energy [6], [7].

When the rapid DC discharge has values over $3 \mathrm{kV}$ and $300 \mathrm{~J}$, the electron is then fully "compressed" into the proton resulting in the synthesis of the neutron (Fig. 3). The point important for this section, is that the neutron cannot be synthesized from a hydrogen gas without the proton and the electron passing thorough the intermediate state of the neutroid (Fig. 4).

It should be indicated that the neutroid cannot exist for quantum mechanics because the lowest possible bound state of a proton and an electron admitted by quantum mechanics occurs at mutual distance of the order of $10^{-8} \mathrm{~cm}$.

However, the neutroid is fully predicted by the covering hadronic mechanics, and in actuality, it is an unavoidable intermediate state prior to the full synthesis of the neutron (Fig. 3). The neutroid is created by the opposing charge and magnetic moment of the proton and electron in singlet coupling that are extremely large at $1 \mathrm{fm}$ mutual distances, plus the contact, non-linear, non-local and non-Hamiltonian interactions that arenumerically stronger than said Coulomb interactions as established in Section 5 of Ref. [6] of 1978 and confirmed thereafter.

According to currently available experimental information, the neutroid is created by a rapid DC discharge in a hydrogen gas when its voltage and energy are below the indicated threshold values of $3 \mathrm{kV}$ and $300 \mathrm{~J}$. Alternatively, it is easy to see that the achievement of the compression of the electron inside the proton (Fig. 3) implies the necessary transition through the configuration of the neutroid (Fig. 4).

An experimentally intriguing aspect is that the neutroid is not generally detected by conventional neutron detectors evidently due to its null spin compared to the spin $1 / 2$ of the neutron. However, the neutroid is a bona fide nucleon, thus causing nuclear transmutations when absorbed by nuclei, as illustrated later on in Section 3.

To the best of our knowledge at this writing, it appears that neutroid irradiation causes nuclear transmutations with ensuing delayed decay that are not generally present following neutron irradiation reported in detail in Refs. [31], [32].

The above studies essentially establish that all the early tests on the neutron synthesis [4], [5] did not actually synthesize the neutron from the hydrogen because there would be the violation of the conservation of the energy since their experimental set up was insufficient to supply the missing $0.782 \mathrm{MeV}$. However, preceding tests [4], [5] produced indeed evidence of nuclear transmutation caused by nucleons that could solely originate from the interior of the reactor, thus confirming the production of neutroids.

In closing, to prevent misinterpretations, we should recall that the synthesis of the neutrons from the hydrogen does indeed exist in nature, despite its incompatibility with quantum mechanics, thus implying the intermediate neutroid state. Consequently, the characteristics of the neutroid, such as its mean life and rest energy, are unsettled at this writing, however its existence is beyond doubt. 


\section{Prediction of Negatively Charged Nucleons and Nuclei}

\subsection{The Prediction of Pseudoprotons}

Despite efforts over three quarters of a century and investments of billions of dollars in the high energy nuclear fusions ("hot fusions"), low energies nuclear reactions ("cold fusions") and other approaches, we have been unable to achieve to date new, clean, industrially viable, nuclear energies. In the author's view. a primary reason for the difficulties to achieve controlled nuclear fusions is the Coulomb repulsion between positively charged nuclei, which repulsion, being inversely proportional to the square of the mutual distance, assumes extremely large values at distances activating attractive nuclear forces (conditions known as the Coulomb barrier).

This occurrence has suggested the studies by the author herein reported essentially based on the search for negatively charged strongly interacting particles (hadrons)and nuclei, since they would be attracted (rather than being repelled) by nuclei, thus eliminating said Coulomb barrier, and permitting the initiation of basically new vistas in the search for clean nuclear energies. In this paper, we report the author's efforts in the search for negatively charged hadrons and nuclei. Following their experimental verifications, applications to new clean nuclear energies will be reported in subsequent works.

It should be also indicated that negatively charged hadrons and nuclei may have additional significant applications, in due time and under sufficient funding, such as the recycling of radioactive nuclear waste via their stimulated decay. medical treatment in lieu of the current use of a proton irradiation, and other possible applications.

An important prediction of the studies here reported, is the existence of a new negatively charged nucleon first introduced by the author in Ref. [39] under the name of pseudoproton denoted with the symbol $\mathrm{p}^{-}$(see also studies [40], [41] and comments in papers [4], [35]).

By recalling the synthesis of the neutron, Eq. (2) the predicted pseudoproton is characterized by the "compression" of the electron, this time, inside the neutron (patent pending), see Fig. 5,

$$
n+e^{-} \rightarrow \bar{p}+v
$$

where the isoelectron is again assumed to have a null total angular momentum (as it is the case for the neutron), resulting in a new particle with the following characteristics: negative elementary charge $q=$ $e$; spin $s=1 / 2$; radius essentially that of the neutron; estimated mean life of $5 s$; and rest energy of the order of that of the neutron (since the electron mass and Coulomb interactions can be ignored in first approximation).

Besides the prediction by hadronic mechanics, an argument supporting the existence of the pseudoproton is the following: incontrovertible evidence in nature establishes that the electron can indeed be "compressed" inside the proton despite its incompatibility with quantum mechanics reviewed in Section 1.1. Then, the characteristics of a second electron "compression" (such as mean life and rest energy) are unsettled at this writing, but not its existence, evidently with a probability smaller than that for the neutron.

\subsection{The Prediction of Protoids}

As indicated in Section 1.2. the neutron synthesis implies the existence of an intermediate state called the neutroid. Along essentially the same lines, the existence of the pseudoproton implies the existence of an intermediate state, here introduced, apparently for the first time, under the name of protoid and symbol $\tilde{p}$ (patent pending), according to the reaction (Fig. 7)

$$
n_{\uparrow}+e_{\downarrow}^{-} \rightarrow \tilde{p},
$$




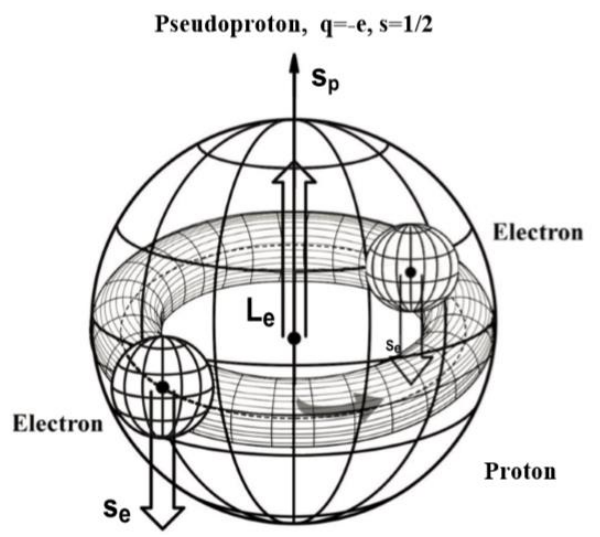

Fig. 5. A schematic view of the synthesis of the negatively charged nucleon known as "Santilli's pseudoproton" [39] via Rutherford's compression of an electron, this time, within the hyperdense neutron.

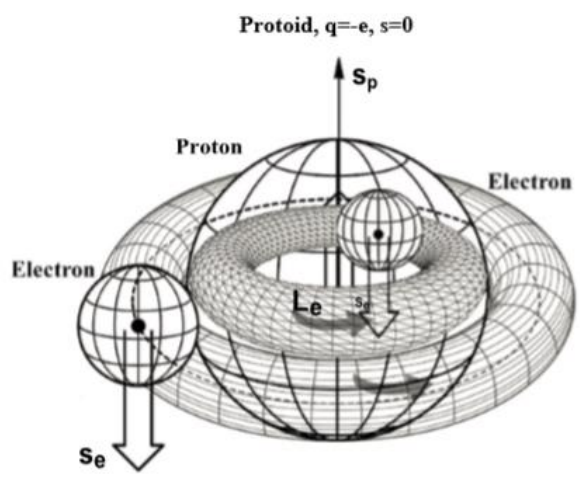

Fig. 6. A schematic view of an intermediate state prior to the full synthesis of the pseudo-proton introduced in this paper for the first time under the name of pseudo-protoid which is given by a bound state in singlet coupling of an electron and a neutron under the strongly attractive contact interactions of isomechanics.

with the following predicted characteristics: negative elementary charge $q=e$; spin $s=0$; mean life predicted to be of about $3 s$; and rest energy of the order of that of the neutron.

An important feature of the protoid is its predicted mean-life, which is expected to be a very large multiple of the mean life of particles produced with accelerator, thus being sufficient for possible practical applications, such as irradiation similar to thoseexpected for the pseudoproton.

\subsection{The Prediction of Iso-Pseudoprotons and Iso-Protoids}

Prediction (8) is based on "Rutherford's compression" of an electron inside the hyper- dense neutron under a sufficiently strong rapid DC discharge or other means, which pre- diction is not unique. In fact, the author achieved in monograph [15] the first known attractive force between identical electrons in singlet valence couplings at short distance. This result was due to the admission of a contact, non-Hamiltonian interaction due to the deep mutual penetration of the wavepackets of the valence electron which, in singlet coupling (only) is so strong to overcome the notorious Coulomb repulsion caused by identical charges. The resulting electron pair was called the isoelectronium with charge $q=-2 e$ and spin $s=0$ because solely derivable via the use of isomathematcs and related isochemistry, and denoted $\left.\left(e_{7}\right), e_{\downarrow}\right)$.

The point important for this paper is that the DC electric arc is not expected to separate into electrons all isoelectronia existing in the original hydrogen molecules. This implies the prediction that isoelectronia are present in the plasma surrounding the DC arc. In turn, this implies the prediction of "Rutherford's 
compression" of the isoelectronium within the hyperdense neutron resulting in a new particle here introduced apparently for the first time under the name of iso-pseudoproton and denoted $\bar{p}$ according to the reaction (Fig. 7)

$$
n+\left(e_{\uparrow}, e_{\downarrow}\right) \rightarrow \bar{p}^{1},
$$

with negative elementary charge $q=e$, spin $\underline{s}=1$ (even though the isoelectronium has zero spin, its constrained orbital motion carries the same spin of the neutron), mass of the order of that of the neutron, and mean life of the order of seconds or fraction thereof.

As it is the case for the pseudoprotons, the configuration of the protoid is (Fig. 6) not unique, since there exist a second configuration, here introduced for the first time under the name of iso-protoid essentially characterized by the fact that the isoelectronium is strongly attracted by protons resulting in a new particle here introduced $\tilde{p}$, according to the reaction

$$
p+\left(e_{\uparrow}, e_{\downarrow}\right) \rightarrow \tilde{p}^{1},
$$

with negative elementary charge $q=e$, spin $s=\underline{1} / 2$ (under the assumption that the orbital motion is in its ground state), and mean life of the order of seconds or fraction thereof.

All composite particles considered so far are expected to be produced by the Directional Neutron Source filled up with a commercially available hydrogen gas of Fig. 12-15, described in detail in Section 4. Possible applications to new nuclear energies are indicated in Section 5.

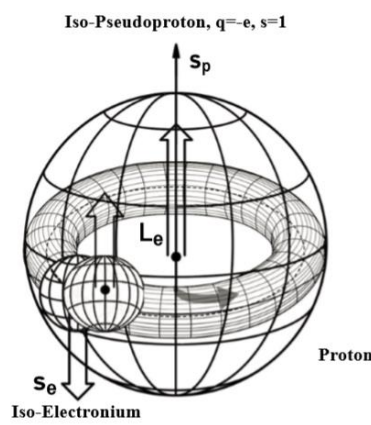

Fig. 7. A schematic view of the alternative synthesis of the pseudoproton introduced in this paper for the first time under the name of "iso-pseudoproton" which is given by Rutherford's compression, this time of a pair of electrons in singlet coupling within the hyperdense proton.

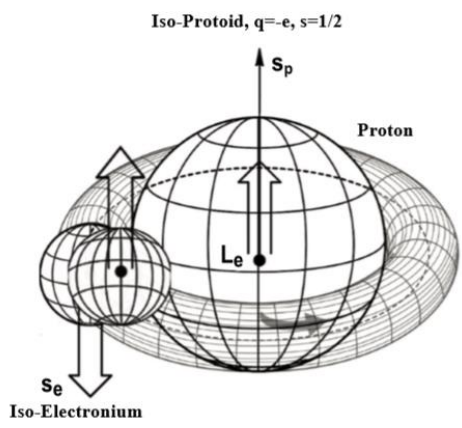

Fig. 8. A schematic view of an intermediate state prior to the full synthesis of the pseudoproton introduced in this paper for the first time under the name of iso-protoid which is given by a bound state of a pair of electron and neutron in singlet coupling under the strongly attractive contact interactions of isomechanics.

\subsection{The Prediction of Pseudo-Deuterons, Deuteroids, et al.}


In a series of paper written in the late 1990's (see Ref. [16] and papers quoted therein), the author achieved the first known, numerically exact and time invariant representation of all characteristics of the deuteron " $d$ ") as a three-body structure composed by two protons and electron verifying the laws of hadronic mechanics according to the reaction (see the conceptual rendering in Fig. 8) based in the following main assumptions:

$$
p_{\uparrow}+e_{\downarrow, J=0}+p_{\uparrow} \rightarrow d
$$

1) The deuteron constituents are not point-like as requested by quantum mechanics, but are extended, thus deformable charge distributions characterized by being isounitary, isoirreducible isorepresentations of the covering Lorentz-Poincare'-Santilli isosymmetry [14], [38] known as isoparticles in general and isoproton and isoelectrons, in particular. The first known numerically exact and time invariant representation of the anomalous magnetic moment of the deuteron then follows from the deformability of its constituents.

2) The deuteron constituents are no longer solely subjected to action-a-distance, potential interactions but, being extended and in conditions of partial mutual penetration, are under potential as well as contact, non-linear, non-local and non-potential interactions solely representable via isomathematics and isomechanics. The admission of the extended character under the most general known forces has then permitted the representation of the stability of the deuteron despite the instability of the neutron (when isolated).

3) Under their extended-deformable character and generalized interactions, conventional treatments of angular momenta and spin have no longer no physical consistency due to the inapplicability of Lie's theory in favor of the covering Lie-Santilli isotheory [7], [11] under which constrained angular momenta within hyperdense hadronic matter can consistently acquire the spin of the environment, resulting in the null total angular momentum of the electron in the synthesis of the neutron, as well as in the synthesis of the deuteron (12). The above new methods have permitted, for the first tie, the representation of the spin, rest energy, charge radius, magnetic moment and the remaining characteristics of the deuteron when its constituents are in their ground state.

The central idea of the studies reported in this paper is "Rutherford compression" of the electron within the hyperdense medium inside the proton, resulting in the synthesis of the neutron. The same idea has then permitted the prediction of the pseudoproton and other negatively charged nucleons. Due to large implications for possible new nuclear energies, in this paper we suggest, apparently for the first time, the study of the additional "Rutherford compression" of electrons, this time, within the protons of the deuteron, resulting in a new negatively charged nucleus hereon called pseudo-deuteron according to the reaction (Fig. 10)

$$
p \uparrow+\left(e_{\downarrow} J=0 e \uparrow, J=0\right)+p \uparrow \rightarrow \bar{d}
$$

where the triplet coupling of the two internal isoelectrons is possible in view of their null total angular momentum.

An alternative possibility that should also be explored in the event of serious commitment to the search for new clean nuclear energies is the compression of an isoelectronium inside the deuteron, resulting again in another negatively charged nucleus here submitted under the name of deuteroid according to the reaction (Fig. 11) 


$$
p_{\uparrow}+e_{\downarrow}, J=0+p_{\uparrow}+\left(e_{\uparrow}, e_{\downarrow}\right) \rightarrow \tilde{p}_{\uparrow}+e_{\downarrow}, J=0+p_{\uparrow} \rightarrow \tilde{d}
$$

Needless to say, unlike structure (12), structures (13) and (14) are predicted to be un- stable. Yet all "Rutherford's compression" are predicted to have mean lives of the order of seconds or fraction thereon, thus being relevant for industrial applications. Note also that all structures (12), (13) and (14) have spin $s=1$, the same mass and charge radius, thus being of rather difficult experimental differentiation among themselves. The predictions of additional negatively charged nuclei, such as the pseudo-tritium, tritoids, etc. is left to interested colleagues.

Note that the pseudo-deuteron and the deuteroid are predicted to be produced by the DNS of Fig. 12 to 15 when filled up with a commercially available deuterium gas at pressure. The efforts for the search of negatively charged nuclei, rather than hadrons is evidently aimed at increasing the possibility for new clean nuclear energies, the recycling of nuclear waste via their stimulated decay, and other applications.

It may be of interest to note that the reduction of the neutron and the deuteron to isoprotons and isoelectrons appears to imply the reduction of all stable matter in the universe to isoprotons and isoelectrons under the covering laws of hadronic mechanics as suggested in refs. [16] and papers quoted therein, and studied in detail in refs.

\subsection{The Prediction of Pseudo-Isotopes}

It is generally believed that all possible nuclear isotopes (also called nuclides), including unstable isotopes created in laboratory, have been tabulated [44]. However, the prediction of new nucleons (neutroids, pseudoprotons and protoids) implies the prediction of three new classes of nuclides formulated in preceding works [30]-[33] here called of pseudo- isotopes (pseudonuclides) with predicted, yet unknown, very short mean life.

To achieve a representation of the pseudonuclides, we use the standard nuclear symbols $(Z, A, J)$ denoting atomic number, atomic mass and spin, respectively. We shall then identify neutrons, neutroids, pseudoprotons and protoids as follows:

$$
\begin{gathered}
n=n(0,1,1 / 2), \\
\tilde{n}=\tilde{n}(0,1,0), \\
\bar{p}=\bar{p}(-1,1,1 / 2), \\
\tilde{p}=\tilde{p}(-1,1,0) .
\end{gathered}
$$

Let us denote a tabulated isotope with the conventional symbol $N(Z, A, J)$ and let us denote pseudo-isotopes with the symbol $\mathrm{N}^{\sim}(\mathrm{Z}, \mathrm{A} . \mathrm{J})$. We then have the following nuclear transmutations:

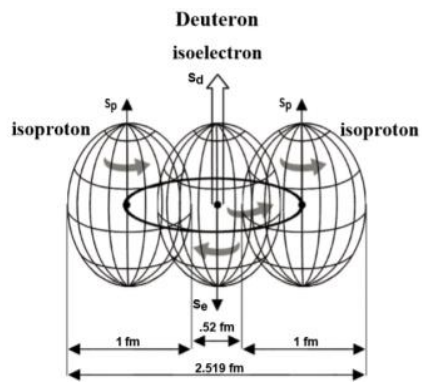

Fig. 9. A schematic view of "Santilli three-body structure of the deuteron" [16] as a bound state of two protons and one electron in conditions mutual penetration, thus acquiring generalized states known as isoprotons and isoelectrons, respectively. 


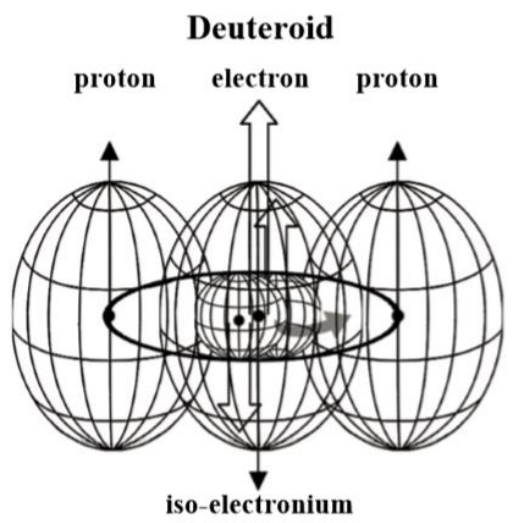

Fig. 10. A schematic view of the possible negatively charged state of the deuteron introduced in this paper for the first time under the name of deuteroids which is given by a bound state of two isoprotons and two isoelectrons under the strongly attractive contact interactions of isomechanics.

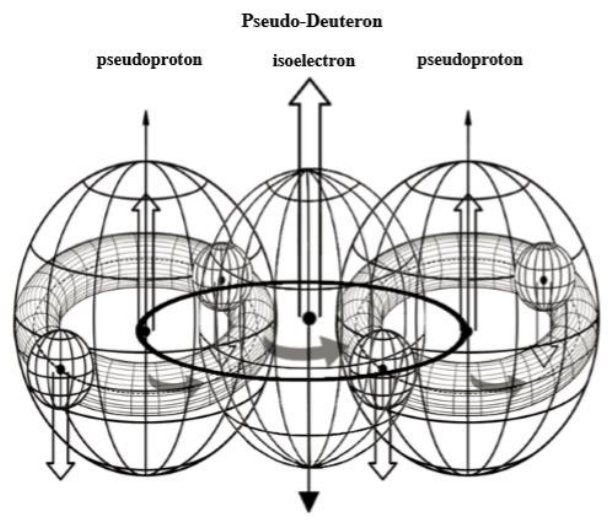

Fig. 11. An alternative negatively charged state of the deuteron introduced in this paper for the first time under the name of iso-pseudo-deuteron" which is given by two isoneutrons and one isoelectron acting as a gluon under the strongly attractive contact interactions of isomechanics.

$$
\begin{aligned}
& N(Z, A, J)+n(0,1,1 / 2) \rightarrow N^{\dagger}(Z, A+1 . J+1 / 2), \\
& N(Z, A, J)+\tilde{n}(0,1,0) ; \rightarrow \tilde{N}(Z, A+1 . J), \\
& N(Z, A, J)+\bar{p}(-1,1,1 / 2) \rightarrow \tilde{N}(Z-1, A+1 . J+1 / 2), \\
& N(Z, A, J)+\tilde{p}(-1.1,0) \rightarrow \tilde{N}(Z-1, A+1 . J),
\end{aligned}
$$

where transmutation (2.4a) yield a tabulated nuclide, while the remaining three transmutations are not expected to be necessarily tabulated, thus yielding pseudonuclides per our definition (patent pending).

A typical illustration of a pseudonuclide is a tabulated nuclide that absorbs a neutroid, resulting in a not necessarily tabulated nuclide, Eq. (2.4b), due to the spin zero of the neutroid. But the neutroid is an intermediate state of the neutron. Therefore, when under the strong interactions existing in the interior of nuclei, the absorbed neutroid is expected to be "completed" into the neutron with the emission of a neutrino decay into a proton with the emission of an electron

$$
\begin{aligned}
& \tilde{n} \rightarrow n+\mu, \\
& \tilde{n} \rightarrow p+e^{-} .
\end{aligned}
$$


The above decays are expected to have short mean life. However, by recalling that very large funds are used in particle physics to produce new particles with mean lives of the order of $10^{-20} \mathrm{~s}$ or so, the expected short mean-life of pseudonuclides is not a scientific reason to deny their possible existence.

Similar situations occur for the creation of pseudonuclides via the absorption of pseudoprotons and protoids.

For clarity, in the next section we shall also use the notations

$$
\begin{gathered}
\beta^{-}=\beta^{-}(0,0,1 / 2), \\
v=v(0,0,0,1 / 2),
\end{gathered}
$$

where the minus sign in the $\beta^{0}$ is intended to recall that its emission implies the increase of $Z$ by one unit.

\subsection{Pseudoprotons or Antiprotons?}

An unavoidable question is the connection between pseudoprotons and the antiprotons [42], [43]. This question was addressed in the original proposal [39] along with the following:

ALTERNATIVE 1. Pseudoprotons and antiprotons are completely different particles, since the former are part of "matter", while the latter belong to "antimatter". This possibility is supported, for instance, by the fact that protoids have spin $s=0$ (Fig. 6), while antiprotons can only have spin $s 1 / 2$.

ALTERNATIVE 2. Pseudoprotons and antiprotons are the same. This possibility is suggested by still unsettled aspects in the current production of antiprotons via scattering of protons on a target (such as the apparent violation of the PCT Theorem). Additionally, matter and antimatter should annihilate into light [42], while experiments on the Bose-Einstein correlation of proton-antiproton scattering establishes the production of a large number of particles and antiparticles [43] generally violating the PCT Theorem and having other insufficiencies. Also, antiprotons claimed to produce a shower of particles (as in the Bose-Einstein correlation), while matter-antimatter annihilation should solely produce light [42].

Hence, Ref. [39] suggests scientific caution and the conduction of additional specific tests establishing the actual matter or antimatter character prior to the final claims of producing true antiprotons.

It should be noted that, in the event antiprotons at CERN, FERMILAB and other national laboratories turn out to be pseudoprotons, said laboratories would acquire for the first time in their history possibilities of important industrial applications for the production if new clean nuclear energies, recycling of nuclear waste via their stimulated decay, and other applications.

\subsection{Etherino or Neutrino?}

The reader should be aware that the transition from the point-like abstraction of pro- tons to their representation as extended and hyperdense particles has a number of rather deep implications for the very foundations of particle physics.

Fermi's hypothesis of the emission of a neutrino in the neutron synthesis (2) was absolutely necessary in the 1940s [3] under the assumption of the exact validity of quantum mechanics for the neutron synthesis.

By contrast, the representation of the proton as an extended and hyperdense particle implies that the total angular momentum of the electron compressed inside the proton is null $J=0$ because of the emergence of the new orbital angular momentum of the electron inside the proton which is constrained to be equal to the proton spin. This implies the confirmation of Rutherford's original conception of the neutron synthesis (1) without any need of the neutrino.

Additionally, in the synthesis of the neutron there is the need for missing $0.872 \mathrm{MeV}$, Eqs. (3), (4) solely needed to reach the neutron rest energy without any consideration for the additional energy needed for the emission of the hypothetical neutrino.

In view of these and other aspects that have remained unsettled for the neutrino hypothesis in about one 
century, following studies 45 through 57 the author submitted in Ref. [58] the hypothesis of the aetherino (from the Latin aether), denoted with the symbol " $a$ ", conceived as alongitudinal impulse, rather than a particle, with charge $q=0$, spin $s=1 / 2$, and rest energy $m=0$, delivering the missing energy to the neutron synthesis from the ether, the environment or other sources according to the reaction

$$
p^{+}+a+e^{-} \rightarrow n \text {. }
$$

where one should note that, as a condition to supply the missing energy, the etherino is on the l.h.s. of the reaction. By contrast, the neutrino cannot be consistently written in the l.h.s. of the reaction to deliver the missing energy, thus requiring the introduction of the new etherino.

We would like to mention that the experimental verification or dismissal of the existence of the pseudoproton has no connection with the open issue of the neutrino vs. the etherino, since the latter merely implies a re-interpretation of available theoretical and experimental data without their numerical changes.

\section{Representative Irradiation Examples}

The main objective of this paper is to provide a report on the ongoing efforts for the experimental verification of the existence of pseudoprotons in view of their rather unique scientific and industrial applications. The experimental methods selected for this paper is the use of stable isotopic analysis of irradiated natural elements by independent qualified laboratories.

In preparation of said analyses, in this section we introduce representative examples of theoretically predicted transmutation of natural elements with neutrons, neutroids, pseudoprotons and protoids. In the next section, we shall compare the theoretical predictions of this section with the results of independent isotope analyses.

The symbols used in this section are those introduced in Section 2.5. The tabulated nuclides quoted in this section are derived from Ref. [44]. Established nuclear transmutations have been derived from Refs. [45].

\subsection{Irradiations of $\mathrm{Ag}(47,107,1 / 2)$}

\subsubsection{Neutron irradiation}

The conventional neutron irradiation of $A g(47,107,1 / 2)$ produces the following nuclear transmutation

$$
\begin{aligned}
& \operatorname{Ag}(47,107,1 / 2)+n(0,1,1 / 2) \rightarrow \tilde{A} g(47,108,1) \rightarrow \\
& \quad \rightarrow C d(48,108,0)[\text { stableisotope }]+e^{-}(-1,0,1 / 2)+v(0,0,1 / 2),
\end{aligned}
$$

resulting in the production of the isotope nuclide $C d(48,108,0)$, as well known [44], [45]. The identification in the irradiated $A g(47,107,1 / 2$ of the stable nuclide $C d(48,108,0)$ sufficiently over the background, would provide an incontrovertible experimental confirmation of the synthesis of the neutrons from the hydrogen by the TEC-DNS of Fig. 5, 6 and 7, in addition to the direct detection of neutrons reported in Refs. [30], [35].

Note that the isotope $\tilde{A} g(47,108,1)$ is a fully tabulated [44], as well known.

\subsubsection{Neutroid irradiation}

$$
\begin{aligned}
& A g(47,107,1 / 2)+\tilde{n}(0,1,0) \rightarrow \tilde{N}(47,108,1 / 2) \rightarrow \\
& \rightarrow C d(48,108,0)[\text { stableisotope }]+e^{-}(-1,0,1 / 2),
\end{aligned}
$$

In this case, Neutroid irradiation produces the same isotope $C d(48,108,0)$ as that predicted by neutron irradiation along decay (3.2.1). Consequently, neutroid irradiation can- not be experimentally distinguished from neutron irradiation via stable isotope analyses and requires different methods, such as that of delayed decays indicated in Section 1.2. 
Note that the intermediate isotope $\mathrm{N}^{\sim}(47,108,1 / 2)$ is not tabulated [44] (because of the anomalous spin $1 / 2$ ), thus being a pseudo-isotope as defined in Section 2.4 .

\subsubsection{Pseudoproton irradiation}

$$
\begin{aligned}
& \operatorname{Ag}(47,107,1 / 2)+\bar{p}(-1,1,1 / 2) \rightarrow \tilde{N}(46,108,1) \\
& \rightarrow \quad \operatorname{Pd}(46,108,0)[\text { stable isotope }]+\gamma(0,0,1),
\end{aligned}
$$

Resulting in the production of the stable isotope $\operatorname{Pd}(46,108,0)$ which is different than the isotope $C d(48,108,0)$ produced by neutron irradiation. Consequently, the identification in the irradiated $A g(47,107$, $1 / 2)$ of $P d(46,108,0)$ (sufficiently over the background) would provide clear experimental evidence on the existence of thepseudoproton.

Note that isotope isotope. $\tilde{N}(46,108,1)$ is not tabulated [44] and therefore, it is a pseudo-isotope

\subsubsection{Protoid irradiation}

$$
\begin{aligned}
& A g(47,107,1 / 2)+\tilde{p}(-1,1,0) \rightarrow \tilde{N}(46,108,1 / 2) \rightarrow \\
& \quad \rightarrow \operatorname{Pd}(46,108,0)[\text { stableisotope }]+v(0,0,1 / 2),
\end{aligned}
$$

Resulting in the same stable isotope $P d(46,108,0)$ as that predicted by pseudoproton irradiation. Consequently, stable isotope analysis does not allow an experimental differentiation between pseudoprotons and then protoids, with resulting need for different methods treated in future works.

Nevertheless the identification of $P d(46,108,0)$ in irradiated $\operatorname{Ag}(47,107,1 / 2)$ would establish the scientifically and industrial most important existence of negatively charged nucleons with mean life sufficient for irradiations and related application, irrespective of whether such particles have spin 1/2 or 0.

Note that the intermediate isotope $\tilde{N}(46,108,1 / 2)$ is also not tabulated [44], thus being a pseudo-isotope.

\subsection{Irradiations of $\operatorname{Ag}(47,109,1 / 2)$.}

\subsubsection{Neutron irradiation}

$$
\begin{aligned}
& A g(47,109,1 / 2)+n(0,1,1 / 2) \rightarrow A g(47,110,1) \rightarrow \\
& \rightarrow C d(48,110,0)[\text { stable isotope }]+e^{-}(-1,0,1 / 2)+v(0,0,1 / 2),
\end{aligned}
$$

Resulting in the stable isotope $C d(48,110,0)$, as well known. Therefore, the identification via stable isotope analysis in the irradiated $A g(47,109,1 / 2)$ of $C d(48,110,0)$ (sufficiently over the background) would provide a second experimental evidence of the synthesis of the neutron from the hydrogen, in addition to the evidence presented in Refs. [34]-[39].

Note that the intermediate isotope $\operatorname{Ag}(47,110,1)$ is fully tabulated, as well known.

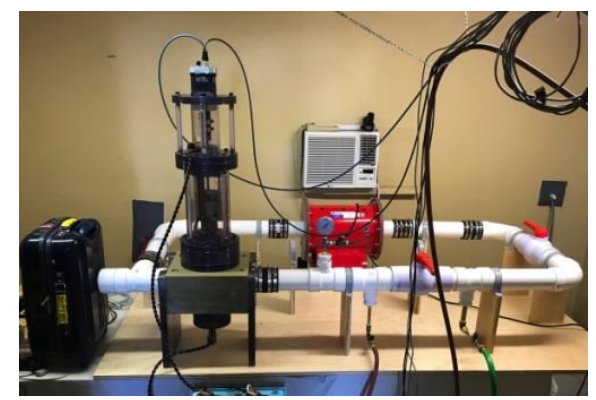

Fig. 12. A picture of the "Directional Neutron Source" (DNS) based on the laboratory synthesis of the neutron from the hydrogen which is in production and sale by Thunder Energies Corporation [46] for the detection of fissionable material that may be concealed in suitcases. 


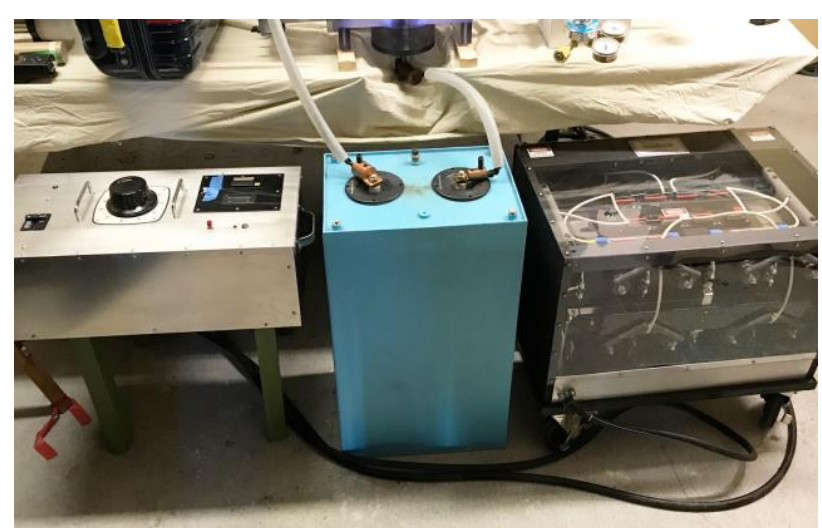

Fig. 13. A picture of the power unit and capacitor of the DNS of Fig. 12.

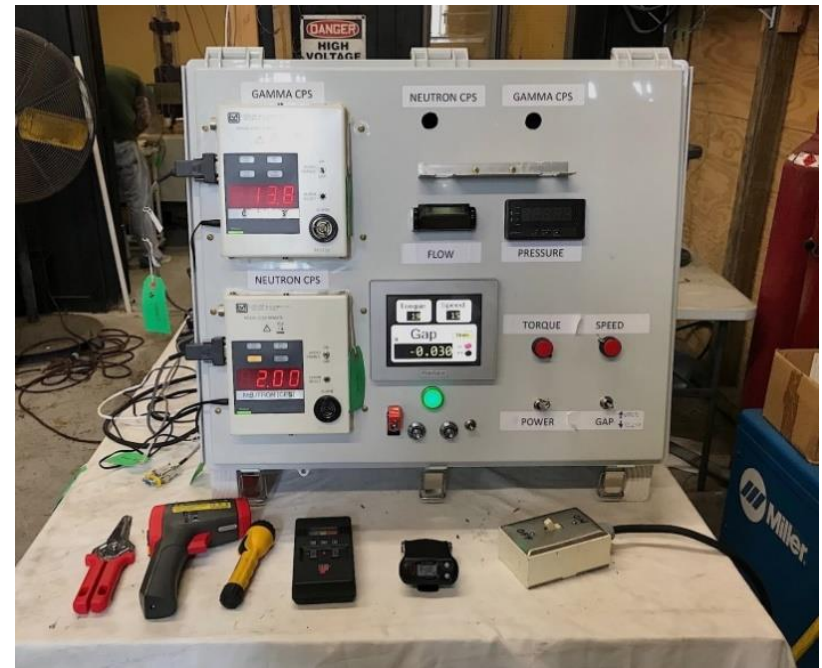

Fig. 14. A picture of a remote monitoring and control station of the DNS of Fig. 12.

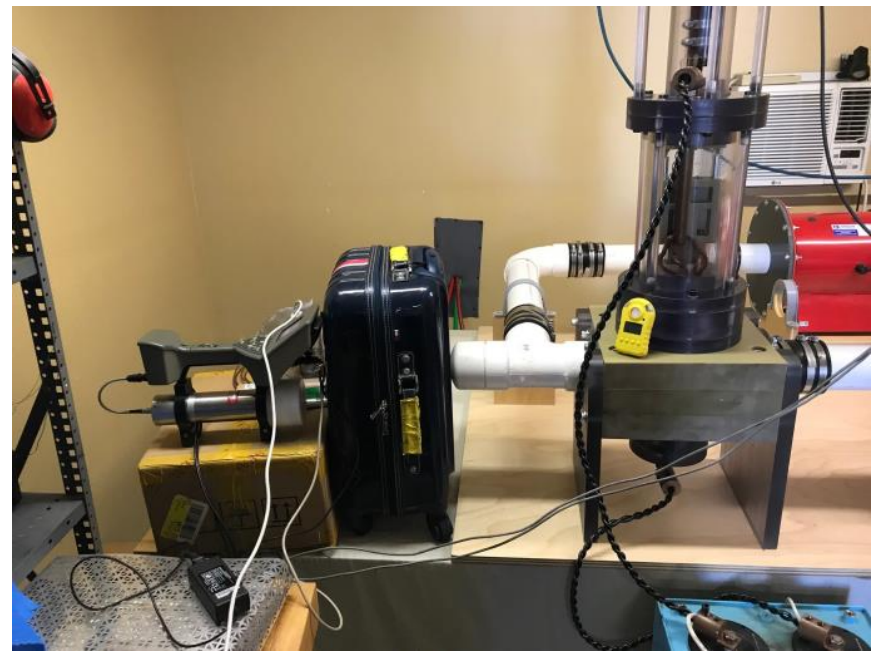

Fig. 15. A picture of detectors capturing the radiation emitted by fissionable material that may be contained in a suitcase when irradiated by a neutron flux.

\subsubsection{Neutroid irradiation}

$$
\begin{aligned}
& A g(47,109,1 / 2)+\tilde{n}(0,1,0) \rightarrow \tilde{N}(47,110,1 / 2) \rightarrow \\
& \rightarrow C d(48,110,0)[\text { stableisotope }]+e^{-}(-1,0,1 / 2),
\end{aligned}
$$


resulting also in the same stable isotope $C d(48,110,0)$ produced by neutron irradiation, thus preventing the use of stable isotope analyses for their differentiation.

Note that the intermediate isotope $\tilde{N}(47,110,1 / 2)$ is not tabulated [44] (again, because of the anomalous values of the spin), thus being a pseudo-isotope.

\subsubsection{Pseudoproton irradiation}

$$
\begin{aligned}
& A g(47,109,1 / 2)+\bar{p}(-1,1,1 / 2) \rightarrow \tilde{N}(46,110,1) \rightarrow \\
& \rightarrow \operatorname{Pd}(46,110,0)[\text { stableisotope }]+\gamma(0,0,1) \gamma(0,0,1),
\end{aligned}
$$

resulting in new stable isotope $P d(46,110,0)$ which is different than the isotope $C d(48,110,0)$ predicted by neutron irradiation, thus being particularly suited for tests via isotopic analyses.

Note that the intermediate isotope $\mathrm{N}^{\sim}(46,110,1)$ is not tabulated, thus being a bona fide pseudo-isotope.

\subsubsection{Protoid irradiation}

$$
\begin{aligned}
& \operatorname{Ag}(47,109,1 / 2)+\mathrm{p}^{\sim}(-1,1,0) \rightarrow \mathrm{N}(46,110,1 / 2) \rightarrow \\
\rightarrow & \operatorname{Pd}(46,110,0)[\text { stableisotope }]+\mathrm{v}(0,0,1 / 2)
\end{aligned} \rightarrow
$$

resulting in the same stable isotope $P d(46,110,0)$ predicted by pseudoproton irradiation. Consequently, the experimental differentiation between pseudoprotons and protoids re- quires methods other than isotopic analyses to be considered in some futurepaper.

Note that $\tilde{N}(46,110,1 / 2)$ is another yet untabulated pseudo-isotope.

\subsection{Irradiations of $\mathrm{Au}(79,197,3 / 2)$.}

\subsubsection{Neutron irradiation}

$$
\begin{gathered}
A u(79,197,3 / 2)+n(0,1,1 / 2) \rightarrow A u(79,198,2) \rightarrow \\
\rightarrow H g(80,198,0)(\text { stableisotope })+e^{-}(-1,0,1 / 2)-v(0.0 .1 / 2) \gamma(0,0,1),
\end{gathered}
$$

resulting in the stable isotope $H g(80,198,0)$, as well known [44], [45]. Consequently, the identification of $H g(80,198,0)$ in irradiated $A u(79,197,3 / 2)$ would provide a third clear experimental confirmation of the synthesis of neutrons from the hydrogen.

\subsubsection{Neutroid irradiation}

The predicted neutroid irradiation is given by

$$
\begin{array}{r}
A u(79,197,3 / 2)+\tilde{n}(0,1,0) \rightarrow \tilde{N}(79,198,3 / 2) \\
\rightarrow H g(80,198,0)+\beta(-1,0,1 / 2)+\gamma(0,0,1),
\end{array} \rightarrow
$$

resulting again the same stable isotope $H g(80,198,0)$ as that produced by neutron irradiation, thus requiring methods other than stable isotope analyses for their differentiation.

Note that the intermediate isotope $\tilde{N}(79,198,3 / 2)$ is not fully tabulated, thus being a pseudo-isotope.

\subsubsection{Pseudoproton irradiation}

$$
\begin{aligned}
& A u(79,197,3 / 2)+\bar{p}(-1,1,1 / 2) \rightarrow \tilde{N}(78,198,2) \rightarrow \\
& \rightarrow \quad \operatorname{Pt}(78,198,0)[\text { stable isotope }]+2 \gamma(0,0,1),
\end{aligned}
$$

resulting again in the stable isotope $P t(78,198,0)$ which is differentthan the isotope $H g(80,198,0)$ produced by neutron irradiation. Therefore, the irradiation of $A u(79,197,3 / 2)$ provides a viable method for the verification of the existence of pseudoprotons.

Note that the intermediate isotope $\tilde{N}(78,198,2)$ is also not tabulated [44], thus being a pseudo-isotope. 


\subsubsection{Protoid irradiation}

$$
\begin{gathered}
A u(79,197,3 / 2)+\tilde{p}(-1,1,0) \rightarrow \tilde{N}(78,198,3 / 2) \rightarrow \vec{\rightarrow} \\
\rightarrow \quad \operatorname{Pt}(78,198,0)[\text { stableisotope }]+\gamma(0,0,1)+v(0,0,1 / 2),
\end{gathered}
$$

resulting in the new stable isotope $P t(78,198,0)$ which is the same as that for predicted by pseudoproton irradiation.

Note that the intermediate isotope $\mathrm{N}^{\sim}(78,198,3 / 2)$ is not tabulated, thus being a pseudo-isotope.

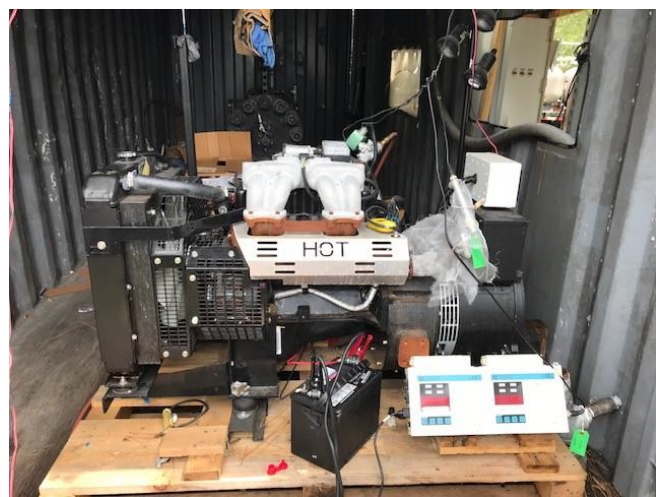

Fig. 16. A picture of the $12 \mathrm{~kW}$ General Motor Electric generator (GMEG) used for the experimental verification of the pseudoproton reported in this paper for the first time.

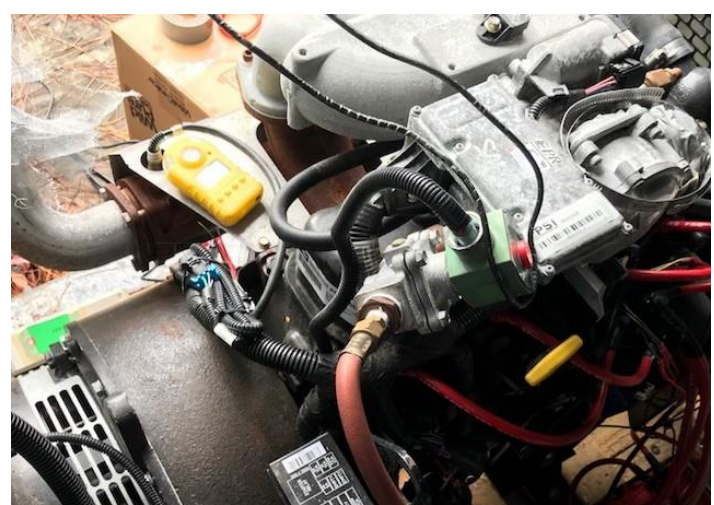

Fig. 17. A picture of the sole feeding of the GMEG of Fig. 16 via a hydrogen gas with a sealed air intake Fig. 18.

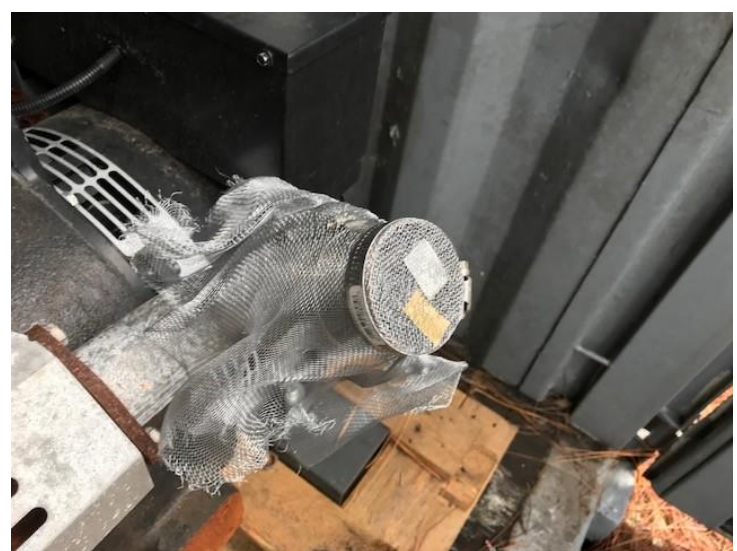

Fig. 18. A picture of the placement of the irradiation of samples of natural Silver and Gold with the exhaust of the GMEG of Fig. 16 when solely fed with a hydrogen gas and operated via its standard crank. 


\subsection{Irradiation of $\mathrm{Cu}(29,65,3 / 2)$}

Among a virtually endless number of additional irradiations, we here present the irradiation of $\mathrm{Cu}(29,65$, $3 / 2$ ) as an example of irradiation not recommended for the verification of existence or dismissal of the pseudoproton.

\subsubsection{Neutron irradiation}

$$
\begin{aligned}
& C u(29,65,3 / 2)+n(0,1,1 / 2) \rightarrow C u(29,66,1)+\gamma(0,0,1) \rightarrow \\
& \rightarrow Z n(30,66,0)+\beta(-1,0,1 / 2)+v(0,0,1 / 2) .
\end{aligned}
$$

\subsubsection{Pseudoproton irradiation}

$$
\begin{gathered}
C u(29,65,3 / 2)+\bar{p}(-1,1,1 / 2) \rightarrow \tilde{N}(28,66,2) \rightarrow \\
\rightarrow N i(28,66,0)+2 \gamma(0,0,1) \rightarrow C u(29,66,1)+\beta(-1,0,1 / 2) \rightarrow \\
\rightarrow Z n(30,66,0)+\beta(-1,0,1 / 2)+v(0,0,1 / 2) .
\end{gathered}
$$

As one can see, the irradiation of $\mathrm{Cu}(29,65,3 / 2)$ via neutrons and pseudoprotons pro- duces the same stable isotope $Z n(30,66,0)$, thus preventing their differentiations.

Interested readers can then work out numerous additional nuclear transmutations of natural elements.

\subsection{Irradiation Summary}

The most important predictions of the above irradiations suitable for test via stable iso- tope analyses are the following:

1) Neutron irradiation of $\operatorname{Ag}(47,107,1 / 2)$ producing the stable isotope $C d(48,108,0)$

2) Pseudoproton irradiation of $A g(47,107,1 / 2)$ producing the different stable iso- tope $\operatorname{Pd}(46,108,0)$

3) Neutron irradiation of $\operatorname{Ag}(47,109,1 / 2)$ producing the stable isotope $C d(48,110,0)$

4) Pseudoproton irradiation of $\operatorname{Ag}(47,109,1 / 2)$ producing the different stable iso- tope $\operatorname{Pd}(46,110,0)$

5) Neutron irradiations of $A u(79,197,3 / 2)$ producing the stable isotope $\operatorname{Hg}(80,198,0)$

6) Pseudoproton irradiations of $A u(79,197,3 / 2)$ producing the different stable iso-tope $P t(78,198,0)$
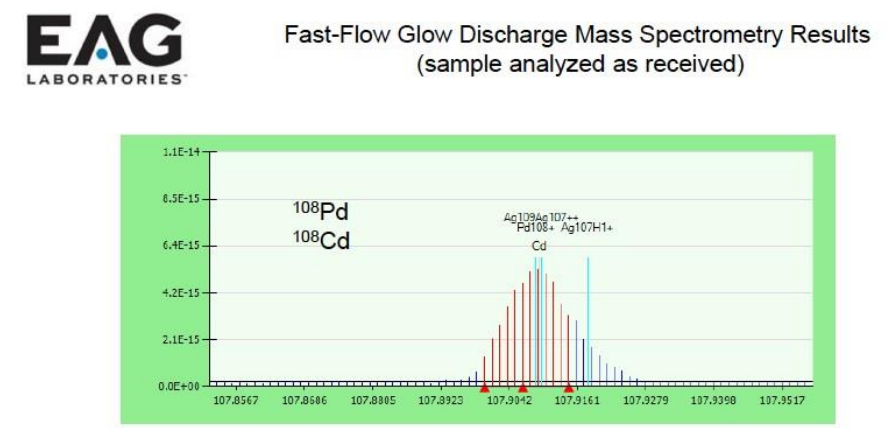

Integrated Counts of ${ }^{108} \mathrm{Pd} /{ }^{108} \mathrm{Cd}=2.7 \mathrm{E} 5$

Method sensitivity:

Pd sensitivity: $6.1 \mathrm{E} 4 \mathrm{cps} / \mathrm{ppm}$

Cd sensitivity: $6.6 \mathrm{E} 4 \mathrm{cps} / \mathrm{ppm}$

\section{EAG Job\# COHVP280; TEC-TNS-GN-01 Ag sample}

Fig. 19. A reproduction of the measurements by the EAG Laboratory of the PD-108 impurity contained in natural silver [53].

\section{Experimental Verifications}




\subsection{Use of a Directional Neutron Source (DNS)}

The prediction of the synthesis of the pseudoproton from a neutron and an electron has been made as a secondary reaction with a lower statistical probability of the synthesis of the neutron from a proton and an electron. Consequently, following studies 45 through 57 the first experimental tests were done via equipment designed for the latter synthesis, namely, via Directional Neutron Source (DNS) as currently available at Thunder Energies Corporation [59] comprising (Fig. 5 and 6, patent pending):

1) A pressure resistant circular loop filled up with commercially available hydrogen which is recirculated by an explosion proof blower inserted in the loop as visible in the back of Fig. 12;

2) An arc control tower visible in the front left of Fig. 12 (shown for the visibility of its structure without the all-encompassing gamma and neutron shields that are visible in [59]) including 3/4" diameter carbon electrodes with their gap submerged in the recirculating hydrogen, the anode being stationary and placed in the lower part of said tower, and the cathode being housed in the upper portion of the tower, its position with respect to the cathode being controlled by a remotely operated stepper motor shown in the top of said tower so as to achieve the desired value of the submerged electrodegap;

3) A rapid discharge capacitor with $80 \mu F$ shown in Fig. 13, plus a specially de- signed high voltage DC power unit with $12 \mathrm{~kW}$ and a variac for the control of said power, shown in the right and left of Fig. 13, respectively;

4) A remote monitoring and control station (Fig. 14) placed at a distance from the DNS with certified lack of radiation including displays for: detected gamma, neutron Counts per Seconds (CPS); pressure and temperature of the recirculating hydrogen; the gap in between the electrodes; alarms for hydrogen leaks and others; as well as controls for the switching on and off of all operations.

5) A number of neutron and gamma detectors not shown in the figures, including: a Ludlum Model 375, dual, neutron and gamma detector with He-3 activation for the neutron counts CPS [47]; a Berkeley Nucleonics SAM 940 dual gamma and neutron detector with Li activation for neutron CPS [48]; an Advacam MiniPix neutron detector with Li activation [49] a Polimaster personal neutron and gamma detector model 1703GNM with Li activation [50] a Berkeley-Nucleonics PalmRad; Aplus dosimeters and other detectors.

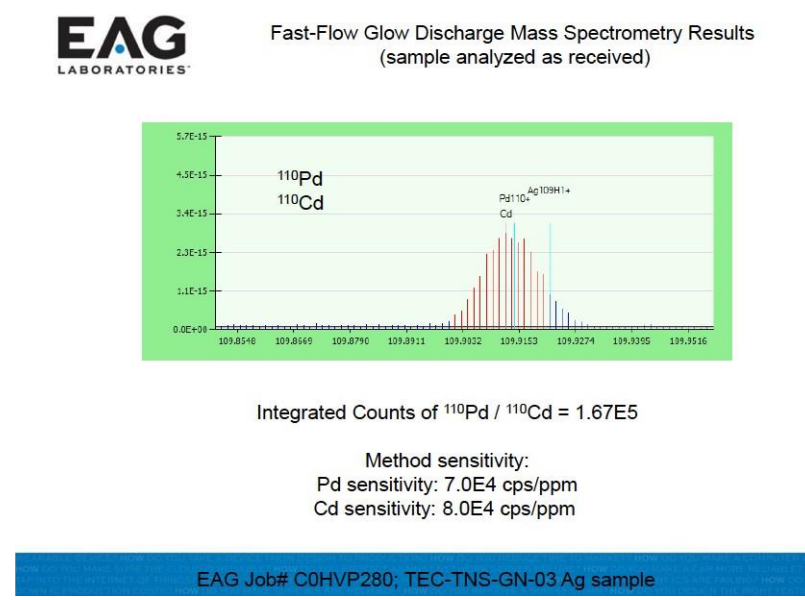

Fig. 20. A reproduction of the measurements by the EAG Laboratory of the PD-110 impurity contained in natural silver [53].

The above DNS is operated as follows: the loop that is originally filled up with air is flushed with hydrogen until the exhaust gas (collected in a tank to avoid air contamination) shows no detectable Oxygen 
under a part per million (ppm) oxygen detector; at that point, the hydrogen in the loop is set at the desired pressure; the gap between the electrodes and the variac of the DC power unit are set at compatible values; the blower and the DC power unit are activated; the power is adjusted for the selected gap via the use of the variac; the DNS is run for the desired, generally clocked period of time at the end of which all systems are disconnected and the gap is reduced to zero to assure that the capacitor is discharged via the electrode short.

A predominant directionality of the emitted neutron flux is assured by recirculation of the hydrogen through the electrode gap; the neutron CPS for a given hydrogen pressure are controlled by the electrode gap and corresponding optimal DC power; and the neutron energy is controlled via the control of the hydrogen pressure and the voltage of the rapid DC discharge.
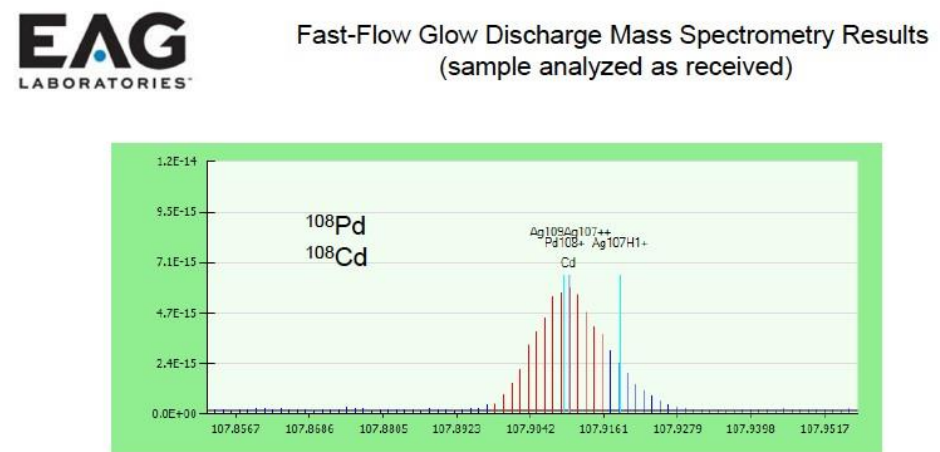

Integrated Counts of ${ }^{108} \mathrm{Pd} /{ }^{108} \mathrm{Cd}=3 \mathrm{E} 5$

Method sensitivity:

Pd sensitivity: $7.0 \mathrm{E} 4 \mathrm{cps} / \mathrm{ppm}$

Cd sensitivity: $8.0 \mathrm{E} 4 \mathrm{cps} / \mathrm{ppm}$

Fig. 21. A reproduction of the measurements by the EAG Laboratory [53] of the Natural Silver irradiated with the exhaust of the GMEG equipment of Fig. 16 showing an excess of Pd-108 over the non-irradiated sample, thus providing an apparent first experimental confirmation of the existence of the pseudoproton.

In the tests herein reported, that were initiated on December 12, 2016, the author used: a commercially available hydrogen gas with $98 \%$ purity, and the DNS was operated with the following data: the hydrogen pressure in the loop was set at $7 \mathrm{psi}$; the electrode gap was initially set at 0.23 with the variac set at the $50 \%$ mark, and then increased to 0.42 " with the variac correspondently set at the $75 \%$ mark; the arc voltage was correspondingly estimated to $4 \mathrm{kV}$ and $6 \mathrm{kV}$; the neutron CPS were correspondingly about 12 and $24 \mathrm{CPS}$; the neutron energy was of the order of $30 \mathrm{~V}$; the rapid DC discharge in the submerged gap of the electrodes was of the order of $1 \mathrm{~Hz}$; and the temperature of the hydrogen as well as of the arc control tower always remained below $120^{\circ} \mathrm{F}$, thus allowing extended tests.

The DNS outlined in the preceding section is ideally suited for the irradiation of samples along the suggestions of Section 3.5, after passing through the electrode gap, thehydrogen gas is expected to carry all composite particles considered in this paper, including neutrons, neutroids, pseudoprotons and protoids. The selected samples can then be easily irradiated by placing them in the white segment of the loop following the electrodes depicted in Fig. 12.

\subsection{Use of a General Motor Electric Generator (GMEG)}

The DNS outlined in the preceding section has been specifically conceived and con- structed to have the 
minimal possible neutron flux and energy sufficient for an effective scan of suitcases in airports for possible concealed fissionable material, as illustrated in Fig. 15. The indicated minimal possible neutron flux and energy are evidently re- quested by federal regulations for the operation of a neutron source in areas exposed to the public [59]. Since the secondary synthesis of pseudoproton is predicted to have a statistical probability at least one tenth that of the primary synthesis of the neutron, the features of the currently available DNS do not appear to be suitable for a clear experimental verification or denial of the existence of the pseudoproton, unless used for extended period of time.
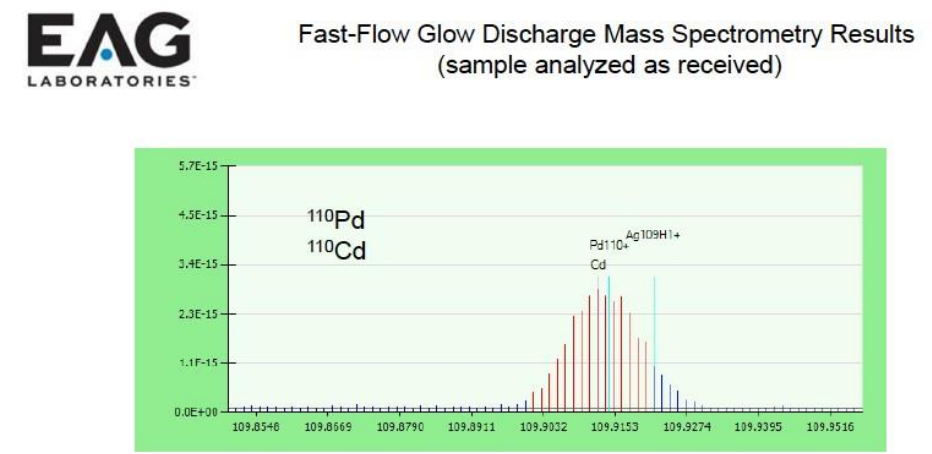

Integrated Counts of ${ }^{110} \mathrm{Pd} /{ }^{110} \mathrm{Cd}=1.67 \mathrm{E} 5$

Method sensitivity:

Pd sensitivity: $7.0 \mathrm{E} 4 \mathrm{cps} / \mathrm{ppm}$

Cd sensitivity: $8.0 \mathrm{E} 4 \mathrm{cps} / \mathrm{ppm}$

Fig. 22. A reproduction of the measurements by the EAG Laboratory [53] of the Natural Silver irradiated with the exhaust of the GMEG equipment of Fig. 16 showing an excess of Pd 110 over the non-irradiated sample, thus providing an apparent second experimental confirmation of the existence of the pseudoproton. A third confirmation is provided by measurements done by the Center for Plasma Mass Spectrometry - Core Research Facility, University of California, Davis [61].

For such a task, a most limiting factor is the rather low pressure of the hydrogen in the recirculating loop (about $7 \mathrm{psi}$ ), compared to the expected need for hydrogen pressure of at least $100 \mathrm{psi}$ so as to achieve a flux of synthesized neutron with at least 140 CPS out of which there are sufficient elements to expect the presence of synthesized pseudoprotons. Unfortunately, the DNS described above, could not be used at 100 psi for safety reasons and its construction for the needed hydrogen pressure is not expected in the near future due to costs.

To solve the above limitations, the author purchased a commercially available General Motor $14 k W$ electric generator model 01705, 3 Liter displacement, 4 cylinders, operating with natural gas depicted in Fig. 16 hereon referred to as the GMEG. The standard ignition of this GMEG was upgraded with a commercially available MSD ignition system used for dragsters (model MSD 8 plus 7805 with Coil Blaster HVC-2, U-Cor), delivering sparks with $45 \mathrm{kV}$ at $235 \mathrm{~J}$, while leaving all remaining features unchanged. This experimental set up allowed the safe achievement of the desired high pressure of the hydrogen, its exposure to an arc with voltage bigger than that of the DNS of Section 4.1, including the possibility of irradiating samples via their exposure of the treated hydrogen coming out of the exhaust.

This upgraded GMEG was used in the tests reported below as follows: the GMEG was placed and operated for safety in the interior of a metal container shown in Fig. 16; the air intake was sealed with a metal cup and related fasteners shown in the right hand side of Fig. 17; in lieu of natural gas, the generator was fed with 
hydrogen (as shown by the red hose in the center of Fig. 17) at 30 psiyielding a total hydrogen pressure at the time of ignition estimated to be of $150 \mathrm{psi}$; the tip of the exhaust was covered with a metal mesh incorporating the selected samples of natural Silver and Gold visible at the left if Fig. 17 as well as in Fig. 18; the generator was started via the use of its standard cranking system operated by a battery connected to an battery charger not shown in the figures; the exhaust gas was collected with a pipe not shown in the figure and compressed in a tank to prevent air contamination; at the end of each test, the hydrogen remaining in the generator was flushed with air.

\subsection{Measurements by the EAG Laboratory}

To confirm the synthesis of the neutron from the hydrogen [34], [35], the author selected the comparative measurements in ppt of $C d(48,108,0)$ and $C d(48,110,0)$ in an non-irradiated and irradiated sample of Natural Silver along proposed tests 3.5.1 and 3.5.3.

Additionally, to initiate the experimental confirmation or denial of the existence of the pseudoproton, the author selected the comparative measurements in ppm of $P d(46,108,0)$ and $P d(46,110,0)$ on samples of non-irradiated and irradiated samples natural Silver also along the same tests 3.5.1 and 3.5.3.

To proceed, the author purchased in early February 2017, two samples of natural Silver $2 \mathrm{~cm}$ square and $2 \mathrm{~mm}$ thick from ESPI Metals [51] with certification of impurities indicated below. One sample was kept away from any irradiation, while the second was exposed to the exhaust of the GMEG shown in Fig. 18 for about 7 minutes of operation at the conditions identified in Section 4.2.

The non-irradiated sample of natural silver was labeled "TEC-TNS-GN-01, Silver un- treated," and kept by the engineer in charge of operating the reactor, always away from the GMEG as well as from the DNS. The irradiated sample was marked "TEC-TNS-GN- 03, Silver pseudoproton active" and placed in contact with the exhaust of the GMEG as shown in Fig. 18, so as to be perpendicular to the hydrogen exhaust. The so labeled and prepared samples were shipped to EAG Laboratory [52] for the above indicated comparative measurements.

The EAG report dated March 7, 2017, EAG Job No C0HVP280, can be downloaded from the link of Ref. [53]. Fig. 19 reproduces page 6 of report [53] on the measurements of 108-Cd and $108-\mathrm{Pd}$ in the non-irradiated sample 101 with sensitivity values

$$
\text { Cd sensitivity : 6. 6E4cps/ppm }
$$

and integrated values

$$
\begin{gathered}
P d \text { sensitivity : } 6.1 \mathrm{EAcps} / \mathrm{ppm} \\
\text { IntegratedCountsof } 108 \mathrm{Pd} / 108 \mathrm{Cd}=2.7 \mathrm{E5}
\end{gathered}
$$

Fig. 20 reproduces page 7 of report [53] on the measurement of 110-Cd and 110-Pd in the non-irradiated sample 101 with sensitivity values

$$
\text { Cd sensitivity: } 6.6 E 4 c p s / p p m
$$

and integrated values

$$
\begin{gathered}
\text { Pd sensitivity : } 6.1 \mathrm{EAcps} / \mathrm{ppm} \\
\text { Integrated Counts of } 110 \mathrm{Pd} / 110 \mathrm{Cd}=1.56 \mathrm{E} 5
\end{gathered}
$$


Fig. 21 reproduces page 10 of report [53] on the measurements of 101-Cd and 108- $\mathrm{Pd}$ in the irradiated sample 103, with sensitivity values

$$
\text { Cd sensitivity: } 8.0 E 4 \mathrm{cps} / \mathrm{ppm}
$$

and integrated values

$$
\text { Pd sensitivity : 7.0E4cps/ppm }
$$

$$
\text { Integrated Counts of } 108 \mathrm{Pd} / 108 \mathrm{Cd}=3 E 5
$$

Finally Fig. 22 reproduces page 11 of report [53] on the measurements of $101-\mathrm{Cd}$ and $110-\mathrm{Pd}$ in the irradiated Silver sample 103, with sensitivity values

$$
\begin{aligned}
& \text { Cd sensitivity : } 8.0 E 4 \mathrm{cps} / \mathrm{ppm} \\
& \text { Pd sensitivity : } 7.0 E 4 \mathrm{cps} / \mathrm{ppm}
\end{aligned}
$$

and integrated values

$$
\text { Integrated Counts of } 110 \mathrm{Pd} / 110 \mathrm{Cd}=1.67 \mathrm{E} 5
$$

As one can see, EAG isotopic measurements [52], [53]provide confirmation of the neutron synthesis from the hydrogen [34], [35], as well as encouraging evidence of the synthesis of pseudoprotons. In fact, the integrated count of $108-\mathrm{Cd}$ and $108-\mathrm{Pd}$ is $3 E 5$ for the irradiated 103 Silver sample as compared to the value $2.7 E 5$ of the non-irradiated sample, with a count increase of $0.30 E 5$ which is significant. For the case of 110 -Cd and 110-Pd we have a total count of $1.67 E 5$ of the irradiated sample 103 compared to the total count of $1.56 E 5$ for the non-irradiated sample, with an increase of $0.11 E 5$. The smallcharacter of the latter value could be explained with the smaller percentage of 101-Pd ( $11.72 \%$ ) compared to that of 108-Pd (26.46\%) in natural Palladium.

On March 7, 2017, EAG Laboratories conducted a second set of measurements [54] in the same samples used for the above first measurements, although measurements [54] were done for different isotopes. Consequently, measurements [54] do not have direct relevance for the aspects studied in this paper.

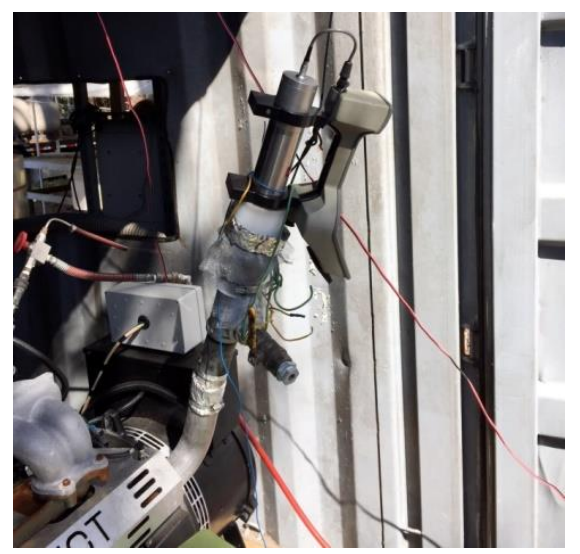

Fig. 23. A picture of the placement of neutron detector sensors directly placed inside the exhaust of the GMEG equipment of Fig. 16 showing the absence of any neutron flux, thus confirming that the excess Pd-108 and Pd-110 detected in measurements [53], [61] can only be caused by a flux of pseudoprotons. 


\subsection{Measurements by the UC-Davis Laboratory}

Following one year of search for a qualified second laboratory willing to confirm or deny measurements [53] done by the EAG Laboratory, the author finally obtained in summer 2018 the cooperation by the Center for Plasma Mass Spectrometry - Core Research Facility, University of California, Davis (hereon referred to "UC-Davis Laboratory" [61]. Following the necessary contractual formalities, in September 2018 the author prepared two samples of natural Silver, the first non-irradiated and the second irradiated with the same reactor GMEG and, with the same operation and for the same duration of time used for the samples tested by the EAG Laboratory; marked them "TEC-18-150, Silver, Untreated," and "TEC-18-151, Silver, Irradiated" and shipped them for comparative spectroscopic analyses of Cd-108, Cd-110, Pd-108, and Pd-110 to the indicated new laboratory.

The UC-Davis Laboratory released on October 10, 2018 the results of their analyses in report [61] with the following summary:

108Pd: The irradiated Ag Sample has an increase in the 108Pd signal. The 108-159Pd signal is practically free of Cd interference.

108Cd: The irradiated Ag Sample has an increase in the 108Cd signal. The majority of the 108-108Cd signal is likely from 108-108Pd interference. The increase in 108-108Cd may be entirely from an increase in 108-108Pd (interference).

110Pd: The irradiated Ag Sample has an increase in the 110Pd signal. The 110-161Pd signal is practically free of Cd interference.

110Cd: The irradiated Ag Sample has an increase in the 110Cd signal. About $2 / 3$ of the 110-110Cd signal is likely from 110-110Pd interference. About $1 / 2$ to $2 / 3$ of the increase in 110-110Cd may be from an increase in 110-110Pd (interference).

As one can see, measurements [61] by the UC-Davis Laboratory confirm measurements [53] done by the EAG Laboratory on similar samples of non-irradiated and irradiated natural Silver via the exhaust of the same GMEG reactor (Fig. 18) for the same duration of time, said reactor being operated under the same specifications of Section 4.2.

Since the sole possible origin of the detected excess transmutation of the 108-Ag and 110-Ag isotopes into 108-Pd and 110-Pd isotopes is that via pseudoproton irradiation (Section 3), the above independent dual confirmation constitutes valid experimental evidence on the existence of the pseudoproton.

In order to confirm the lack of measurable excess presence of $108-\mathrm{Cd}$ and $110--\mathrm{Cd}$ isotopes stated in Measurements [61], following the reception of the latter, the author and his technicians (see the Acknowledgments) conducted systematic scans from October 15 to 20, 2018, of the exhaust of the GMEG reactor with all available neutron detectors, with sensors at times directly inserted in the exhaust as shown in Fig. 23.

The latter scans confirmed the absence of any detectable flux of neutrons released in the exhaust of the GMEG reactor during its operation as per Section 4.2. This was expected because, being neutral, neutrons are notoriously penetrating, thus being absorbed by the engine casing, and cannot follow the tortuous path of the exhaust manifold while the pseudoproton, being charged, have no other choice than that of following said tortuous path.

By recalling that excess $108-\mathrm{Cd}$ and $110-\mathrm{Cd}$ can be solely produced via neutron irradiation of natural silver, in view of the notorious difficulties in distinguishing between 108-Cd and 108-Pt, and between 110 $\mathrm{Cd}$ and $110 \mathrm{Pd}$ due to their identical masses, the confirmation of the absence of neutron irradiation, with consequential absence of 108-Cd and 110-Cd isotopes constitutes an additional experimental confirmation of measurements [61] on the existence of the pseudoproton.

The author additionally prepared samples of non-irradiated and irradiated natural Gold that were preliminarily analyzed by the UC-Davis Laboratory jointly with the Silver sample [61], although with 
unsettled results due to the use of equations for the resolution of an expected 198-Hg and 198-Pt interference without the knowledge of the absence of excess 198-Hg isotopes in the irradiated sample due to the absence of neutron irradiation necessary for its production.

\subsection{Measurements for Irradiation with the DNS}

The author regrets being unable to report meaningful measurements on irradiation via the DNS reactor of Section 4.1. A Silver sample with said irradiation was prepared in March 19, 2017, marked TEC-TNS-27 and shipped to the EAG Laboratory with the re- quest of conducting comparative measurements with the same methods as that of the pre- ceding analyses [53]. Unfortunately, the analyst selected a different, laser-based method and released on 3/24/17 report [55] showing a decrease of the intensity of the laser beam in the transition from the non-irradiated to the irradiated samples, thus preventing its meaningful use. In fact, as one can see in report [55], the c/s of all isotopes Cd-108, Cd- 110, Pd-108 and Pd-110 decrease in the transition from the TEC-TNS-101 (non-irradiated) to TEC-TNS-103 (GMEG irradiated) and from TEC-TNS-101 to TEC-TNS-27 (DNS irradiated) which decreases are a physical impossibility.

\section{Concluding Remarks}

In a series of works [6]-[38] initiated in the late 1970s at Harvard University under DOE support, the author presented mathematical, theoretical and experimental studies on the synthesis of the neutron from the hydrogen via "Rutherford compression" of the electron within the hyperdense proton as occurring in the core of stars.

The same studies imply the existence of: the neutroid [26], which is a neutral new nucleon with spin $s=$ 0 expected to be an intermediate state prior to the synthesis of the neutron (Fig. 3, 4); the pseudoproton [39], which is a negatively charged new nucleon with spin $s=1 / 2$ expected from a secondary "Rutherford compression" of the electron, this time, within the hyperdense neutron; and the protoid, which is a negatively charged new nucleon with spin $s=0$, presented in this paper apparently for the first time, which is expected to be an intermediate state prior to the full synthesis of the pseudoproton (Fig. 5, 6).

By recalling that the synthesis of the neutron (when isolated) has a mean life of the order of 15 minutes, all the above new nucleons are predicted to have mean lives of the order of seconds or fractions thereof, thus allowing the study of possible industrial applications. All new negatively charged nucleons are predicted to be produced via secondary processes in reactors containing a hydrogen gas at pressure traversed by a submerged special forms of rapid DC discharge.

In this paper, we initiate a systematic search of possible negatively charged nucleons and nuclei; we present two different reactors suitable for the primary synthesis of neutrons from a commercially available hydrogen gas, that are expected to yield a secondary flux of neutroids, pseudoprotons and protoids, the first reactor being a Directional Neutron Source (DNS) [46] (Section 4.1 and Fig. 12-15) and the second reactor being an electric generator (GMEG) solely operated with hydrogen without air intake (Section 4.2 and Fig. 16-18); we show that neutron and neutroid irradiations of natural Silver produce the same Cd-108 and Cd-110 stable isotopes while the pseudoproton and protoid irradiations of natural Silver produce the same Pd-108 and Pd-110 stable isotopes; and we initiate the expectedly laborious experimental verification or denial of the existence of pseudoprotons and protoids.

For the latter scope, we present, apparently for the first time, comparative spectroscopic measurements [53] done in March 2017 by the EAG Laboratory [53] of two samples of natural Silver, the first non-irradiated and the second irradiated with the GMEC reactor of Section 4.2, showing a clear increase of Cd-108, Cd-110, Pd-108 and Pd-110 in the irradiated sample (Fig. 19-22); we additionally present comparative spectroscopic analyses [61] done in October 2018 by the Center for Plasma Mass Spectrometry 
- Core Research Facility, University of California, Davis [61] confirming the increase of Cd-108, Cd-110, Pd-108 and Pd-110 stable isotopes in a sample of Natural Silver also irradiated with the GMEG reactor of Section 4.2; we indicate the inability with available detectors to distinguish between $\mathrm{Cd}$ and $\mathrm{Pd}$ isotopes when having the same number of nucleons, as well as the inability to distinguish between neutrons or neutroids and pseudoprotons or protoids; and we present arguments encouraging the continuation of the measurements,

e.g., for irradiation with the DNS reactor of Section 4.1 which is expected to produce a flux of all nucleons here considered with progressively decreasing statistical probabilities.

A primary motivation for the studies here considered is that, being negatively charged, pseudoprotons and protoids are attracted, rather than being repelled, by nuclei, thus allowing the possible resolution of the historical Coulomb repulsion between nuclei that has prevented the achievement to date of industrially viable new nuclear energies after efforts for three quarter of a century at low and high energies and billions of dollars of investments.

In order to illustrate this possibility, the use of the irradiations of Section 3 readily allows the prediction that: pseudoproton and protoid irradiation of Si-28 yields the stable isotope Al-29 with the release of 8.337 $\mathrm{MeV}$; the same irradiation of C-40 yields the stable isotope $\mathrm{K}-41$ with the release of $8.383 \mathrm{MeV}$; and the same irradiation of Fe-54 yields the stable isotope $\mathrm{Mn}-55$ with the release of $8.347 \mathrm{MeV}$. It is also intriguing to see that the neutron and neutroid irradiation of the same natural elements Si-28, C-40 and Fe-54 yields similar stable isotopes with essentially the same positive energy output.

Most importantly, the above nuclear transmutations emit no harmful radiations and release no radioactive waste. Also important is to note that each of the transmutations triggered by neutron/neutroids and by pseudoprotons/protoids yields about 6.7-times the energy needed for the nucleon synthesis identified in Eqs. (3), (4). Finally, one should note that the difference between neutrons and neutroids as well as that between pseudo- protons and protoids has no industrial relevance due to the identity of the related nuclear transmutations.

In consideration of all the above, it appears advisable to test the industrial production of possible, new, clean, nuclear energies since the irradiation via a flux of neutrons, neutroids, pseudoprotons and protoids appearsto transmute a selectednumber oflight, stableand natural elements into light, stable and natural elements with a smaller mass without the emission of harmful radiation and without the release of radioactive waste.

An additional possible industrial application of pseudoprotons and protoids is their use for the stimulated decay of radioactive waste from current nuclear power plants. As it is well known, nuclear waste constituents, such as Cs-137, Pu-239, Am-241, and other are large, naturally unstable nuclei. Therefore, they must admit a mechanism triggering their stimulated decay which is prohibited by quantum mechanics but fully admitted by the covering hadronic mechanics due to its representation of the irreversibility of the process [16]. In fact, it is possible to show that, in the event said nuclei are hit by a sufficiently dense flux of sufficiently energetic pseudoprotons or protoids, they must decay due to their excess of positive charges and other reasons.

We should also mention the use of a directional flux of neutral and charged nucleons for the detection of precious metals in mining operations which is under study by Thunder Energy Corporation [46].

The success of the indicated industrial possibilities depends not only on properfunding and time, but also and perhaps primarily by the capability of the scientific community to admit the impossibility of achieving new clean nuclear energies via 20th century sciences, allow due scientific process on new sciences without non-scientific interferences, and admit a responsibility for the increasingly alarming climatic events.

\section{Acknowledgments}

Thanks are due to Jan Rak, Simone Beghella-Bartoli, Anil A. Bhalekar, Jeremy Dunning-Davies, Max 
Fomitchev-Zamilov, Richard Norman, and other colleagues for a critical inspection of the paper and invaluable comments. Special thanks are due to Brian Buckley, Scott Wainwright, Geraldo Dieppa Jr, Ben DeVries and other technicians for invaluable assistance in the preparation of the two identical sets of samples used by the EAG Laboratory and the UC-Davis Laboratory, as well as for the crucial verification of the lack of neutron flux in the exhaust of Fig. 18. Additional thanks are due to Carla Santilli and Sherri Stone for the linguistic control of the manuscript.t.

\section{References}

[1] Rutherford, H. (1920). Proc. Roy. Soc. A, 97, 374.

[2] Chadwick, J. (1932). Proc. Roy. Soc. A, 136, 692.

[3] Fermi, E. (1949). Nuclear Physics. University of Chicago Press.

[4] Norman, R. (2017). Neutron/Neutroid synthesis from an arc of current in hydrogen gas: experimental verification and theoretical implications. Quantum Matter.

[5] Borghi, C., Giori, C. C., \& Dall'Olio, A. Communications of CENUFPE, 8, 25.

[6] Santilli, R. M. (1978). Need of subjecting to an experimental verification the validity within a hadron of Einstein special relativity and Pauli exclusion principle. Hadronic J., 1, 574-901.

[7] Santilli, R. M. Foundation of Theoretical Mechanics, Volume I [3a] and II [3b]. Springer-Verlag, Heidelberg, Germany.

[8] Santilli, R. M. (1993). Isonumbers and genonumbers of dimensions 1, 2, 4, 8, their isoduals and pseudoduals, and "hidden numbers" of dimension 3, 5, 6, 7. Algebras, Groups and Geometries, 10, 273.

[9] Santilli, R. M. (1996). Nonlocal-integral isotopies of differential calculus, mechanics and geometries. Isotopies of Contemporary Mathematical Structures, Rendiconti Circolo Matematico Palermo, 42, 7-82.

[10] Jiang, C.-X. (2001). Foundations of Santilli Isonumber Theory. International Academic Press.

[11] Sourlas, D. S., \& Tsagas, G. T. (1993). Mathematical Foundation of the Lie-Santilli Theory. Ukraine Academy of Sciences.

[12] Raul, M. F. G., \& Juan, N. V. (2003). Studies on the tsagas-sourlas-santilli isotopology. Algebras, Groups and Geometries, 20, 1.

[13] Georgiev, S. (2015). Foundations of the IsoDifferential Calculus, Volumes, I, II, III, IV, V,and VI. Nova Scientific Publisher.

[14] Santilli, R. M. (1995). Elements of Hadronic Mechanics, Vol. I and Vol. II [15b]. Academy of Sciences, Kiev.

[15] Santilli, R. M. (2001). Foundations of Hadronic Chemistry, with Applications to New Clean Energies and Fuels. Kluwer Academic Publishers. Russian translation by A. K. Aringazin.

[16] Santilli, R. M. (1998). The Physics of New Clean Energies and Fuels According to Hadronic Mechanics. Special volume of the Journal of New Energy, 318.

[17] Santilli, R. M. (1997). Relativistic hadronic mechanics: nonunitary, axiom-preserving completion of relativistic quantum mechanics. Found. Phys., 27, 625-729.

[18] Santilli, R. M. (1991). Isotopic Generalizations of Galilei and Einstein Relativities, Vol. I [12a] and Vol. II [12b]. Hadronic Press.

[19] Santilli, R. M. (2008). Hadronic Mathematics, Mechanics and Chemistry, Volumes I to V. International Academic Press.

[20] Santilli, R. M. An introduction to new sciences for a new era. Clifford Analysis, Clifford Algebras and their Applications.

[21] Aringazin, A., et al. (1991). Santillis Lie-Isotopic Generalization of Galilei and Einstein Relativities. Kostakaris Publishers, Athens, Greece.

[22] Lohmus, J., Paal, E., \& Sorgsepp, L. (1994). Non-associative Algebrasin Physics. Hadronic Press, Palm Harbor. 
[23] Kadeisvili, J. V. (1997). Santillis Isotopies of Contemporary Algebras, Geometries and Relativities. Ukraine Academy of Sciences.

[24] Davvaz, B. (2007). Hyperings Theory and Applications. International Academic Press.

[25] Raul, M. F. G., \& Juan, N. V. (2015). Fundamentos de la isdotopia de santilli. English translations Algebras, Groups and Geometries, 32, 135-308.

[26] Santilli, R. M. (1990). Apparent consistency of Rutherford's hypothesis on the neutron as a compressed hydrogen atom. Hadronic J., 13, 513.

[27] Santilli, R. M. (1992). Apparent consistency of Rutherford's hypothesis on the neutron structure via the hadronic generalization of quantum mechanics - I: Nonrelativistic treatment. ICTP Communication IC/91/47.

[28] Santilli, R. M. (1992). ICTP Communication IC/91/47.

[29] Santilli, R. M. (1993). Recent theoretical and experimental evidence on the synthesis of the neutron, Communication of the Joint Institute for Nuclear Research, Dubna, Russia, No. E4-93-252.

[30] Santilli, R. M. (1995). Recent theoretical and experimental evidence on the synthesis of the neutron, communication of the JINR, Dubna, Russia, No. E4-93-252. Chinese J. System Eng. and Electr., 6, 177.

[31] Santilli, R. M. (2006). Apparent confirmation of Don Borghi's experiment on the synthesis of neutrons.

[32] Santilli, R. M. (2007). Apparent confirmation of Don Borghi's experiment on the laboratory synthesis of neutrons from protons and electrons. Hadronic J., 30, 29.

[33] Santilli, R. M. New structure model of hadrons, nuclei and molecules. Confirmation of Don Borghi's Experiment on the Synthesis of Neutrons from Protons and Electrons.

[34] Santilli, R. M., \& Nas, A. (2014). Confirmation of the laboratory synthesis of neutrons from a hydrogen gas. Journal of Computational Methods in Sciences and Eng.

[35] Richard, N., Anil, A. B., et al. (2017). Experimental confirmation of the synthesis of neutrons and neutroids from a hydrogen gas. American Journal of Modern Physics, 6(4-1), 85-104.

[36] Kadeisvili, V.(2008). The rutherford-santilli neutron. Hadronic J., 31(1), 1-125.

[37] Burande, C. S. (2016). Santilli synthesis of the neutron according to hadronic mechanics. American Journal of Modern Physics, 5(2-1), 17-36.

[38] Gandzha, I., \& Kadeisvili, J. (2011). New Sciences for a New Era: Mathematical, Physical and Chemical Discoveries of Ruggero Maria Santilli. Sankata Printing Press, Nepal.

[39] Santilli, R. M. (2014). The hypothesis of the pseudoproton. Appendix A of the Paper Clifford Analysis, Clifford Algebras and Their Applications, 3, 1-26.

[40] Santilli, R. M. (2015). Apparent nuclear transmutations without neutron emission triggered by pseudoprotons. American Journal of Modern Physics, 4, 15-18.

[41] Otto, H. V. (2015). Possibilities for the detection of Santilli's neutroids and pseudo-protons. American Journal of Modern Physics.

[42] Santilli, R. M. (2006). Isodual Theory of Antimatter with Applications to Antigravity, Grand Unification and Cosmology. Springer.

[43] ATLAS Collaboration. Retrieved from the website: https://arxiv.org/abs/1502.07947

[44] Table of Nuclides. Retrieved from the website: http://atom.kaeri.re.kr:8080/ton/index.html

[45] Tabulated nuclear decays. Retrieved from the website: http://www4vip.inl.gov/gammaray/catalogs/pdf/gecat.pdf

[46] Thunder Energies Corporation, Directional Neutron Source. Retrieved from the website: http://thunder-energies.com/docs/TEC-DNS-3Za.pdf

[47] Ludlum Measurements. Retrieved from the website: http://ludlums.com/Ludlum neutron-gamma detector model 375 
[48] BNC Berkeley Nucleonics Corp. Retrieved from the website: http://www.berkeleynucleonics.com/model-940

[49] ADVACAM. Retrieved from the website: http://advacam.com/camera/minipix

[50] Polimaster. Retrieved from the website: http://www.polimaster.com/company/profile/

[51] ESPI Metals. Retrieved from the website: http://www.espimetals.com/index.php

[52] EAG Laboratories website: Retrieved from the website: https://www.eag.com/

[53] EAG Laboratories report No. EAG Job No COHVP28. Retrieved from the website: http://thunder-energies.com/docs/eag-first-report.pdf

[54] EAG Laboratories second report. Retrieved from the website: http://thunder-energies.com/docs/eag-second-report.pdf

[55] EAG Laboratories third report. Retrieved from the website: http://thunder-energies.com/docs/eag-third-report.xlsx

[56] Bhalekar, A. A., \& Santilli, R. M. (2016). Exact and invariant representation of nuclear magnetic moments and spins according to hadronic mechanics. American Journal of Modern Physics, 5(2-1), $56-118$

[57] Bhalekar, A. A., \& Santilli, R. M. Reduction of stable matter in the universe to isoprotons and isoelectrons, I: Foundations; II: Configurations of stable nuclei; III: Energy levels of stable nuclei to appear.

[58] Santilli, R. M. (2007). The etherino and/or the Neutrino Hypothesis? Found. Phys., 37, 670.

[59] Thunder Energies Corporation, Directional Neutron Source model TEC-DNS-3Z. Retrieved from the website: http://thunder-energies.com/docs/TEC-DNS-3Z.pdf

[60] Center for Plasma Mass Spectrometry - Core Research Facility, University of California, Davis. Retrieved from the website: http://icpms.ucdavis.edu/

[61] Laboratory Report, Center for Plasma Mass Spectrometry. Core Research Facility, University of California, Davis.

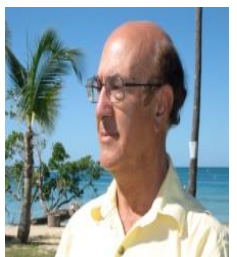

Ruggero M. Santilli obtained his Ph. D. from the University of Torino, Italy; he then and emigrated to the U.S.A. where he was in the Faculty of the University of Miami, Boston University, MIT, and Harvard University under financial support from NASA, UFOSR and DOE; he is the author of 325 papers published in refereed journals and twenty post Ph. D. monographs in mathematics, physics and chemistry; he is internationally known for the discovery of hadronic mathematics, physics, and chemistry and their applications to new clean energies; that have been the subject of over 50 international conferences; he is the editor of numerous scientific journals. Since 2002, Prof. Santilli is the founder and chief scientist of two U. S. publicly traded companies Magnegas Corporation (stock symbol MNGA) and Thunder Energies Corporation ( stock symbol TRNRG) developing cutting edge new technologies; he has received numerous awards including the 2007 Mediterranean Prize, and has been knighted twice with the title of Sir, a first time by the republic of San Marino in 2011 and a second time by the President of Italy, Sergio Mattarella, in June 2018. For details, please see the Biographical Notes http://www.i-b-r.org/Dr-R-M-Santilli-Bio-1-10-18.pdf. 